\title{
Los micromamíferos (Eulipotyphla, Chiroptera, Rodentia y Lagomorpha) del yacimiento del Pleistoceno Superior de la cueva de El Sidrón (Asturias)
}

\section{The micromammals (Eulipotyphla, Chiroptera, Rodentia and Lagomorpha) from the Late Pleistocene site of the El Sidrón cave (Asturias)}

\author{
C. Sesé ${ }^{1}$, M. de la Rasilla ${ }^{2}$ y E. Duarte Matías $^{2}$ \\ ${ }^{1}$ Departamento de Paleobiología, Museo Nacional de Ciencias Naturales, C.S.I.C., C/ José Gutiérrez Abascal 2, 28006, \\ Madrid, España. Email: c.sese@mncn.csic.es; ORCID ID: http://orcid.org/0000-0002-6833-1901 \\ ${ }^{2}$ Área de Prehistoria, Departamento de Historia, Facultad de Filosofía y Letras, Universidad de Oviedo, C/ Amparo Pedregal, \\ s/n, 33011 Oviedo, España. Email: mrasilla@uniovi.es, UO159121@uniovi.es; ORCID ID: http://orcid.org/0000-0002-5505- \\ 0625, http://orcid.org/0000-0003-2767-7049
}

A la memoria de Javier Fortea y Manuel Hoyos

\begin{abstract}
RESUMEN
En el yacimiento del Pleistoceno Superior de la cueva de El Sidrón, con una datación de $\sim 49.000$ ka, conocido por sus numerosos restos fósiles de neandertales, se han recuperado restos de micromamíferos cuyo estudio detallado se realiza en este trabajo. La asociación determinada es la siguiente: Sorex araneus-Sorex coronatus, Neomys cf. fodiens, Talpa sp., Rhinolophus euryale-Rhinolophus mehelyi, Marmota cf. marmota, Eliomys quercinus, Glis glis, Arvicola terrestris, Chionomys nivalis, Microtus arvalis-Microtus agrestis, Microtus lusitanicus, Microtus oeconomus, Clethrionomys glareolus, Apodemus sylvaticus-Apodemus flavicollis y Oryctolagus cuniculus. Estos taxones están presentes en la fauna actual de Asturias y de la región cantábrica excepto Microtus oeconomus que desapareció de la península ibérica en tiempos históricos y actualmente está en regiones euroasiáticas más septentrionales. La fauna de micromamíferos en conjunto parece indicar un medio predominantemente de espacios abiertos, generalmente de praderas de cierta humedad y desarrollo de la vegetación a nivel del suelo, aunque también habría zonas boscosas o arboladas de cierta entidad y algún curso de agua. La presencia, aunque con pocos restos, de algunas especies termófilas, y la ausencia de especies indicadores de clima frío, parecen indicar que el clima durante la formación de los depósitos fosilíferos del yacimiento sería relativamente templado y húmedo, probablemente similar al actual de la zona.
\end{abstract}

Palabras clave: Micromamíferos; Pleistoceno Superior; Región cantábrica; Paleoecología; Paleoclimatología

Recibido el 22 de enero de 2018; Aceptado el 11 de abril de 2018; Publicado online el 15 de junio de 2018

Citation / Cómo citar este artículo: Carmen Sesé et al., (2018). Los micromamíferos (Eulipotyphla, Chiroptera, Rodentia y Lagomorpha) del yacimiento del Pleistoceno Superior de la cueva de El Sidrón (Asturias). Estudios Geológicos 74(1): e76. http://doi.org/10.3989/ egeol.43122.481

Copyright: (C) 2018 CSIC. This is an open-access article distributed under the terms of the Creative Commons Attribution-Non 4.0 International License. 


\begin{abstract}
In the Late Pleistocene site of El Sidrón cave, with a date of $\sim 49,000$ ky, known for its numerous fossil remains of Neanderthals, some remains of micromammals were found, whose detailed study is carried out in this work. The determined faunal association is the following: Sorex araneus-Sorex coronatus, Neomys cf. fodiens, Talpa sp., Rhinolophus euryale-Rhinolophus mehelyi, Marmota cf. marmota, Eliomys quercinus, Glis glis, Arvicola terrestris, Chionomys nivalis, Microtus arvalis-Microtus agrestis, Microtus lusitanicus, Microtus oeconomus, Clethrionomys glareolus, Apodemus sylvaticus-Apodemus flavicollis and Oryctolagus cuniculus. These taxa are present in the current fauna of Asturias and Cantabrian Region except for Microtus oeconomus that disappeared from the Iberian Peninsula in historical times and is currently in northernmost Eurasian regions. The fauna of micromammals as a whole seems to indicate a predominantly open space environment, generally of certain moisture meadows and vegetation development at ground level, although there would also be wooded areas of some entity and some watercourse. The presence, although with few remains, of some thermophilic species, and the absence of cold climate indicator species, seem to indicate that the climate during the formation of the fossiliferous deposits would be relatively temperate and humid, probably similar to the current one in the area.
\end{abstract}

Keywords: Micromammals; Late Pleistocene, Cantabrian Region; Paleoecology; Paleoclimatology.

\section{Introducción}

La cueva de El Sidrón está en el concejo asturiano de Piloña, situado entre la mar y la montaña en el llamado Surco Oviedo-Infiesto. La Sierra del Sueve al noreste, el Monte Cayón al suroeste y, más al sur, el Río Piloña y los Picos de Europa enmarcan el sistema kárstico que se formó en la intersección de unas areniscas y unos conglomerados terciarios. Los regatos Pandu y Gatu han formado la cavidad al circular por su interior, con la incorporación en la zona del Abrigo de La Cabañina de otro regato, La Infiesta, para desembocar al final en el río Borines. La cueva tiene un desarrollo total de $\sim 3.700 \mathrm{~m}$. La entrada principal, denominada La Tumba, se encuentra a 167,89 m.s.n.m., tiene una orientación a poniente y está a unos $15 \mathrm{~km}$ en línea recta de la costa (Fig. 1).

El sitio era conocido desde antiguo, pero el hallazgo en 1994 de unas mandíbulas humanas en su interior, inició un largo proceso, primero judicial, luego administrativo y, finalmente, científico, que ha situado este lugar en los foros nacionales e internacionales (Cañaveras et al., 2018; Fortea et al., 2003, 2007, 2009; Lalueza et al., 2012a, 2012b; Rasilla et al., 2011, 2013; Rosas et al., 2006, 2012, 2013, 2015; Sánchez-Moral et al., 2007). La excavación arqueológica y paleontológica en la Galería del Osario comenzó en 2000 y terminó en 2014 al haberse levantado todo el depósito que contenía el registro arqueológico y antropológico. Tras las investigaciones multidisciplinares llevadas a cabo, y en curso, se cuenta con algo más de 2.500 restos fósiles neandertales en un razonable estado de conservación y unos 400 útiles líticos hechos en sílex de Piloña y cuarcita típicamente musterienses; así como con una pequeña muestra de arte parietal del Paleolítico Superior en la Galería de las Pinturas (Duarte et al., 2016; Fortea et al., 2010; Rasilla et al., 2011; Santamaría, 2006, 2012; Santamaría et al., 2010, 2011; Tarriño et al., 2013).

El modelo de relleno sedimentario propuesto se organiza a partir de varios procesos de alta o baja energía (Fig. 2). Dada la complejidad de la serie deposicional se fueron abriendo sondeos en diferentes puntos de la galería para comprobar, finalmente, que la unidad sedimentaria donde se encuentran los fósiles humanos y la industria lítica llegó desde el techo de la misma a partir de las cuadrículas de la banda 10 extendiéndose hasta la banda 4 (sectores 2 y 3) (Fig. 1) (Cañaveras et al., 2011, 2018; Sánchez-Moral et al., 2007; Santos, 2012a, 2012b; Silva et al., 2011). En ese sentido, la relativamente escasa fauna de macromamíferos hallada, tanto en la Galería del Osario como en otras galerías de la cavidad, no tiene relación directa con los restos neandertales y está compuesta principalmente de ciervo, bóvido (Bos/Bison), rebeco, caballo, lobo y oso (Rosas et al., 2011: 147-148, 180-181).

La utilización de distintos métodos de datación $\left({ }^{14} \mathrm{CAMS}, \mathrm{AAR} / \mathrm{AARD}, \mathrm{ESR}, \mathrm{OSL}, \mathrm{U} / \mathrm{Th}\right.$, Paleomagnetismo) ha ofrecido una información cronológica contrastada de los restos humanos y arqueológicos recuperados. Las dataciones radiométricas sitúan dichos restos en el $\sim 99.000$ ka sin calibrar (Torres et al., 2010; Wood et al., 2013). 

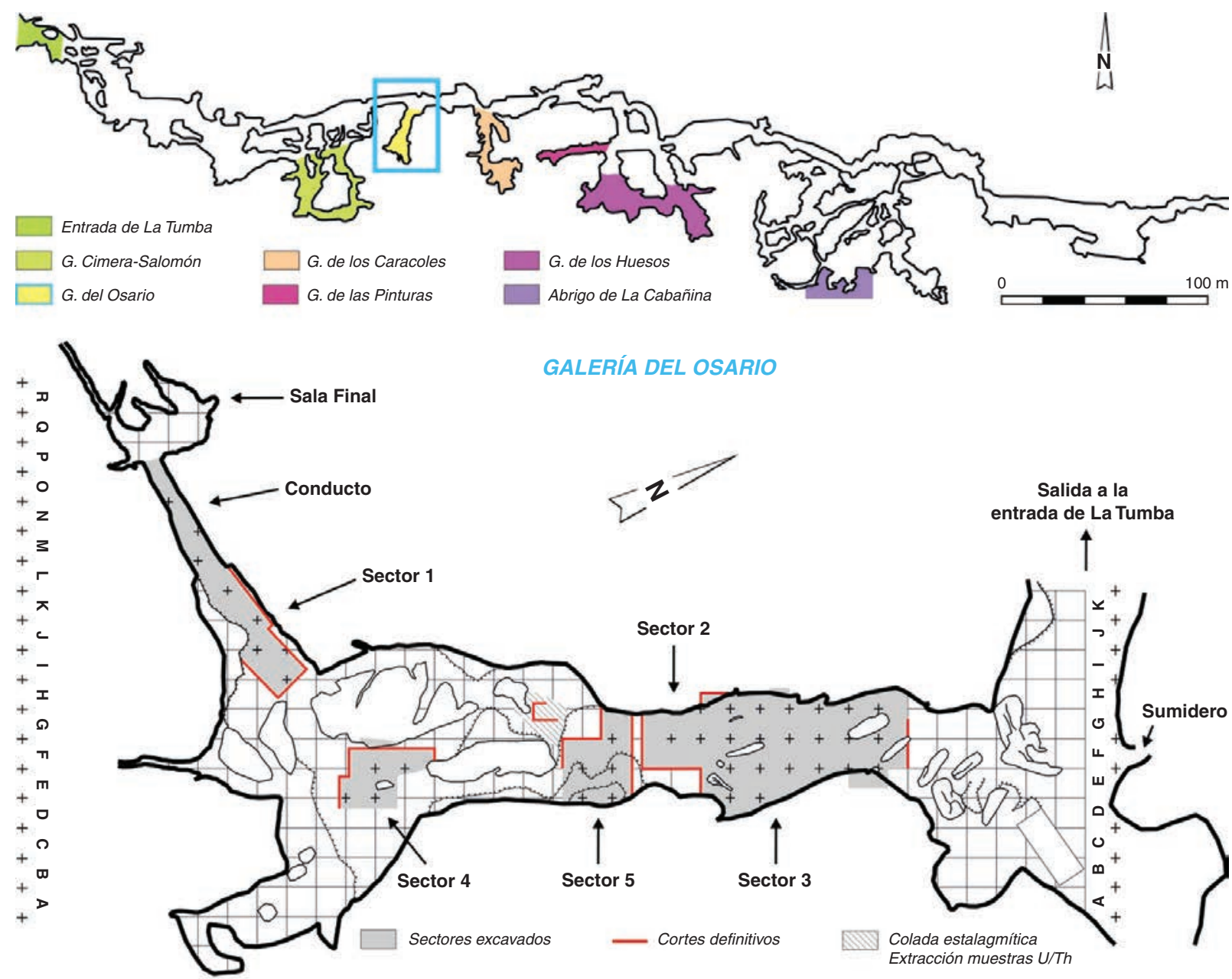

$\begin{array}{llllllllllllllllllllllllllllllllll}32 & 31 & 30 & 29 & 28 & 27 & 26 & 25 & 24 & 23 & 22 & 21 & 20 & 19 & 18 & 17 & 16 & 15 & 14 & 13 & 12 & 11 & 10 & 9 & 8 & 7 & 6 & 5 & 4 & 3 & 2 & 1\end{array}$

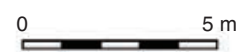

CUEVA DE EL SIDRON. PILOÑA. ASTURIAS

Fig. 1.-Arriba: Planta y principales zonas de intervención arqueológica en el sistema kárstico de El Sidrón. Abajo: Planta y zonas de excavación de la Galería del Osario. Dibujo: E. Duarte y D. Santamaría.

Los micromamíferos, cuyo estudio paleontológico detallado se presenta por primera vez en este trabajo, fueron objeto de un trabajo preliminar de divulgación en Sesé (2011) formando parte del libro editado por Rasilla et al. (2011) sobre los hallazgos más relevantes realizados en la Cueva de El Sidrón. La fauna determinada en dicho trabajo por Sesé (2011) fue la siguiente: Sorex sp., Neomys fodiens, Talpa sp., Rhinolophus euryale-Rhinolophus mehelyi, cf. Marmota marmota, Glis glis, Apodemus sp., Arvicola terrestris, Microtus arvalis-Microtus agrestis, Microtus lusitanicus y Oryctolagus cuniculus.
El presente estudio de la fauna de micromamíferos viene a completar la información multidisciplinar sobre el yacimiento de El Sidrón.

\section{Metodología}

El sedimento del yacimiento se lavó y cribó con agua utilizando dos cribas: la de abajo con una luz de malla de $1,41 \mathrm{~mm}$, y la de encima con una luz de malla de $2,38 \mathrm{~mm}$.

La determinación taxonómica de los diferentes taxones de micromamíferos se ha realizado sobre 


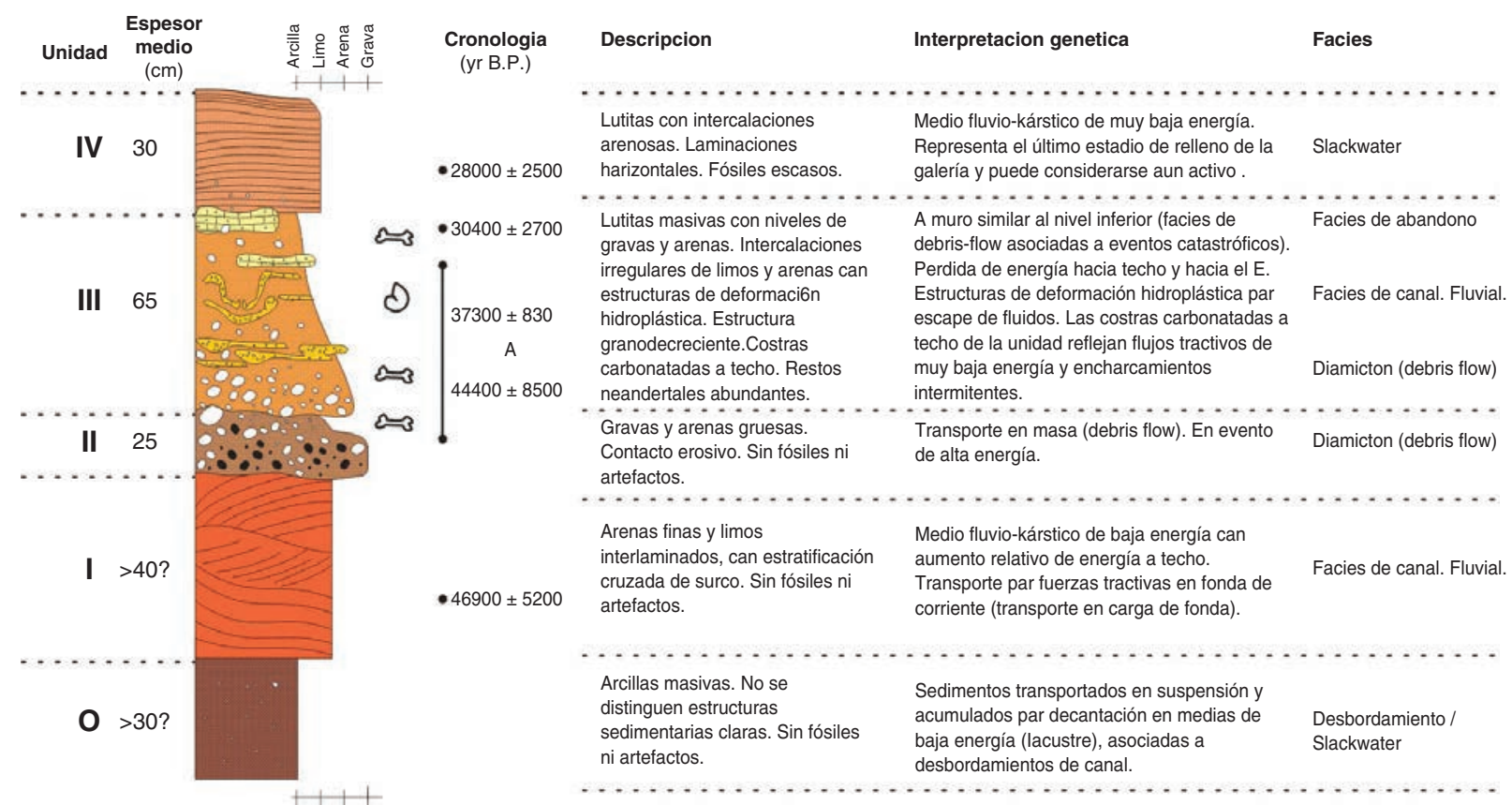

Fig. 2.-Columna litoestratigráfica tipo representativa del relleno de la zona central de la Galería del Osario; con descripción somera de las principales unidades sedimentarias definidas y su interpretación genética (Cañaveras et al., 2011, e. p.).

el siguiente material: en los arvicolinos, los $\mathrm{m} 1$ que son los que permiten determinaciones taxonómicas precisas; en los esciúridos, glíridos y murinos todo el material dentario; en los lagomorfos los p3; en los erinaceomorfos y soricomorfos las mandíbulas, maxilares superiores y dentición; en el topo, además de los restos mandibulares, maxilares y dentición, algunos huesos del esqueleto postcraneal como cúbitos y húmeros. En el texto se dice mandíbula y maxilar superior, derecho o izquierdo, y no hemimandíbula y hemimaxilar superior derecho o izquierdo para simplificar.

Las abreviaturas utilizadas son: Incisivo (I), Canino (C), Premolar deciduo inferior (d); premolar inferior/superior $(\mathrm{p} / \mathrm{P})$; molar inferior/superior $(\mathrm{m} / \mathrm{M})$; mandíbula (man.); maxilar superior (max. sup.); Longitud (L); Anchura (A); derecho (der.); izquierdo (izq.); fragmento (fr.); indeterminado (indet.); Número Mínimo de Individuos (NMI); Museo Nacional de Ciencias Naturales (MNCN).

Se ha utilizado el NMI para comparar la abundancia relativa de los diferentes taxones teniendo en cuenta la pieza identificable taxonómicamente más abundante entre derechas e izquierdas, adultos y juveniles. Con respecto a los arvicolinos del género Arvicola, se ha reconocido una sola especie en el yacimiento teniendo en cuenta los $\mathrm{m} 1$, que son las piezas que permiten su identificación taxonómica específica. Por ello, en aquellos niveles en los que no se ha encontrado esta pieza dentaria sino otras piezas craneales y dentarias que se reconocen por su talla y especial morfología como pertenecientes al género Arvicola, para obtener el NMI se han tenido en cuenta este otro tipo de molares distintos al $\mathrm{m} 1$; en estos casos y bajo el epígrafe de "Otro tipo de molar", el número de molares y el NMI resultante se ponen entre paréntesis para indicar que se ha obtenido de otra forma a la habitual. En el apartado del material correspondiente a cada taxón se da por zonas y niveles el número de piezas que permiten su identificación, así como el NMI obtenido.

Las medidas se han realizado con una lupa Nikon Measuroscope 10 5x que lleva incorporado un micrómetro de $0,025 \mathrm{~mm}$ de precisión y se expresan en milímetros (mm). Los dientes de micromamíferos y mandíbulas de soricomorfos se han dibujado utilizando un microscopio Nikon SMZ-10 40x que tiene una cámara clara acoplada. Los dibujos fueron retocados utilizando el programa CorelDRAW. Todo el material 
de El Sidrón, cuando finalice su estudio, quedará depositado en el Museo Arqueológico de Asturias.

Se han manejado los siguiente trabajos: para la distribución geográfica actual en España y Europa, y los hábitats de las especies que se registran en el yacimiento: Blanco (1998), Pokines (1998), Palomo \& Gisbert (2002) y Mitchell-Jones et al. (1999); para la distribución biostratigráfica y geográfica de dichas especies en el Pleistoceno y su interpretación paleoclimática se han utilizado los de Pemán (1985), Sesé (1994 y 2005), Sesé \& Sevilla (1996), Kowalski (2001), Arribas (2004), López-García (2008) y García-Ibaibarriaga (2015); y para las consideraciones tafonómicas se han tenido en cuenta los trabajos de Chaline et al. (1974), Andrews (1990) y Fernández-Jalvo et al. (2016).

\section{Paleontología Sistemática}

Familia Soricidae FISCHER, 1814

Subfamilia Soricinae FISCHER, 1814

Sorex araneus LINNAEUS, 1758 - Sorex coronatus MILLET, 1828

(Fig. 3: a)
Material por niveles:

- Zona F9, Nivel III: 1 fr. man. izq. con p4-m1m2-fr. m3; NMI: 1

- Zona F21, Nivel III: 1 fr. man. der. con I-m1-m2, rama acescente y proceso articular; NMI: 1

- Zona M27/28, Nivel I: 1 fr. cráneo con bóveda craneal, max. izq. con P3-P4-M1-M2 y max. der. con P3-P4-M1-M2; NMI: 1

NMI TOTAL: 3

\section{Comentarios:}

Los dientes presentan la pigmentación en rojo de la parte superior de las cúspides y crestas que las reúnen característica del género Sorex. De talla similar a Sorex araneus y Sorex coronatus, el material es muy escaso como para poder atribuirlo con seguridad a una de estas dos especies cuya distinción es difícil especialmente cuando, como en este caso, hay poco material dentario, por lo que en la literatura científica el material de este tipo de soricinos suele citarse bajo la denominación conjunta de ambas especies en muchos yacimientos.

S. araneus se distribuye actualmente por casi toda Europa desde el norte de la península ibérica hasta

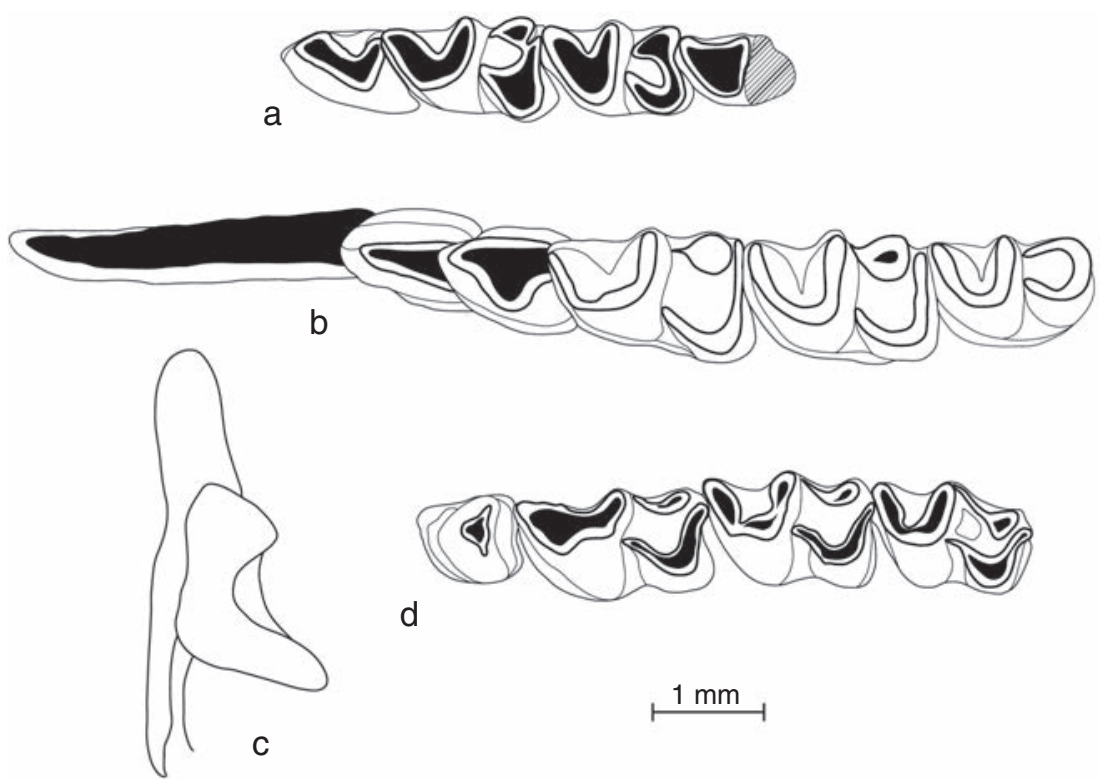

Fig. 3.-Dibujos de material craneal y dentario del yacimiento de la cueva de El Sidrón: Sorex araneus - Sorex coronatus: a) Mand. izq. con serie dentaria: p4-m1-m2-fr. m3 (Sidrón 2004; F9; 1; 4; III; 23; Caja 28); Neomys cf. fodiens: Mand. izq.: b) Serie dentaria: I-p3p4-m1-m2-m3); c) Cóndilo articular en vista posterior (Sidrón 2009; E4; 9; 2a , III; 1267; Caja 61); Rhinolophus euryale - Rhinolophus mehelyi: d) Mand. izq. con serie dentaria: p4-m1-m2-m3 (Sidrón 2005; E10; 3; 7a; III; 13; Caja 24). 
Siberia. En España solo se encuentra en el norte de Cataluña: en parte del Pirineo, Prepirineo y región oriental húmeda del Montseny. Estas poblaciones y las de la vertiente francesa del Pirineo están aisladas de las del resto de Europa, supuestamente debido a la retracción que sufrió la especie al final de la última glaciación, ya que durante el Pleistoceno Medio y Pleistoceno Superior estuvo mucho más extendida en España y Europa (Arribas, 2004; Palomo \& Gisbert, 2002). Vive en bosques caducifolios, mixtos y de coníferas, y en pastizales húmedos. Habita en ambientes de gran humedad ambiental, en zonas con una pluviosidad superior a los $800 \mathrm{~mm}$ anuales.

$S$. coronatus vive en el norte de la península ibérica, en Francia y en el occidente de Alemania, Suiza y Austria. Requiere ambientes y suelos húmedos con bastante vegetación. Se encuentra en bosques caducifolios, pastizales y riberas de los ríos, desde el nivel del mar hasta los $2.200 \mathrm{~m}$ de altitud. En Asturias y en general en la cornisa cantábrica solo se encuentra actualmente $S$. coronatus, aunque hay muchas citas de $S$. araneus en yacimientos del Pleistoceno Superior de esta última región.

$S$. araneus parece que estuvo ampliamente distribuida durante el Pleistoceno Medio y Pleistoceno Superior, y sufrió una notable reducción durante este último periodo, mientras $S$. coronatus se expandía en dicho periodo a sus expensas.

A diferencia de otros yacimientos de edades similares a El Sidrón, como los niveles auriñacienses y musterienses de la cueva de El Castillo (Sesé, 2017a) en los que $S$. coronatus es relativamente abundante (hasta el 19,3\% del NMI total de micromamíferos del nivel Auriñaciense 18c), en El Sidrón la representación de este soricino es muy escasa tal como se ha dicho, con un NMI de 3 y un porcentaje del NMI total en todo el yacimiento del 3,6\% (véase la Tabla 1).

Neomys cf. fodiens (PENNANT, 1771)

(Fig. 3: b-c)

Material por niveles:

- Zona E4/F7, Nivel III: 1 fr. man. der. con m1$\mathrm{m} 2-\mathrm{m} 3$, rama ascendente y proceso articular; 1 fr. man. izq. con I-p3-p4-m1-m2-m3, rama ascendente y proceso articular; NMI: 1 NMI TOTAL: 1

\section{Comentarios:}

Este material presenta las características propias del género Neomys: las cúspides dentarias están pigmentadas en rojo, el incisivo inferior no presenta cúspides, y el cóndilo articular de la mandíbula tiene las dos facetas articulares notablemente separadas por un área interarticular estrecha, la faceta superior es relativamente corta y la inferior alargada lingualmente. El carácter que permite distinguir a Neomys fodiens de Neomys anomalus CABRERA, 1907 es la altura coronoidea de la mandíbula según varios autores. Según Saint Girons (1972) (en: Pemán, 1983; p. 119 ) en $N$. fodiens varía entre $4,3 \mathrm{~mm}$ y $5,3 \mathrm{~mm}$, y en $N$. anomalus entre $3,8 \mathrm{~mm}$ y 4,6 mm; según Pemán (1983) en $N$. fodiens es superior a $5,1 \mathrm{~mm}$ y en $N$. anomalus inferior a $4,7 \mathrm{~mm}$; según Chaline et al. (1974) en $N$. fodiens varía entre $4,4 \mathrm{~mm}$ y $5,3 \mathrm{~mm}$ y en $N$. anomalus entre $3,9 \mathrm{~mm}$ y $4,4 \mathrm{~mm}$; y según Palomo \& Gisbert (2002) en $N$. fodiens es superior a $4,7 \mathrm{~mm}$ y en $N$. anomalus inferior a $4,8 \mathrm{~mm}$. En las dos mandíbulas de El Sidrón los valores de la altura coronoidea: 4, $93 \mathrm{~mm}$ y 4,75 $\mathrm{mm}$ respectivamente, están más próximos a los de $N$. fodiens que a los de $N$. anomalus, aunque el material es tan escaso que realizamos con reservas su atribución a la primera especie como Neomys cf. fodiens.

En la península ibérica durante el Pleistoceno Superior $N$. fodiens solo se ha citado en yacimientos de la franja norte peninsular (Sesé, 1994 y 2005; Arribas, 2004; López-García, 2008). Actualmente se extiende desde el norte de la península ibérica hasta el lago Baikal en Siberia. Es la musaraña más acuática: está ligada a zonas litorales y ribereñas de lagos y ríos de aguas oxigenadas, aunque también puede vivir en zonas húmedas de bosques, praderas y pantanos.

Familia Talpidae FISCHER, 1814 Subfamilia Talpinae FISCHER, 1814

\section{Talpa sp.}

\section{Material por niveles:}

- Zona G8, Nivel III: 1 fr. man. izq. sin dientes con alveolos de dos primeros premolares y molares; NMI: 1

- Zona H8, Nivel IV: 1 fr. húmero der.; NMI: 1

- Zona F21/22, Nivel III: 1 cúbito der.; 1 fr. de cúbito der.; 1 húmero der.; NMI: 2 


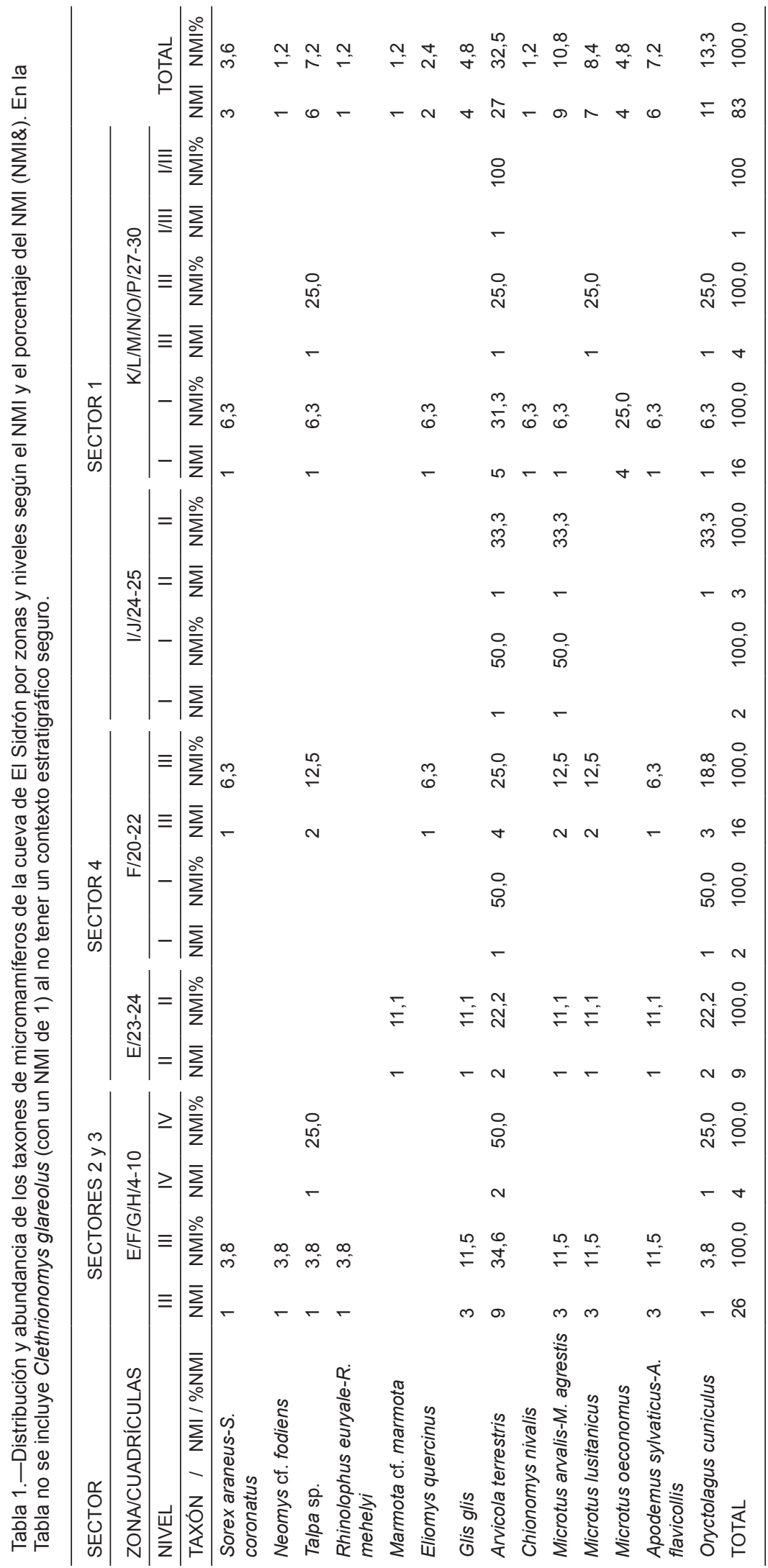


- Zona M27/28, Nivel I: 1 fr. man. der. sin dientes con alveolos de los dientes, rama ascendente y proceso articular: NMI: 1

- Zona N28/M28, Nivel III: 1 fr. man. izq. con cuatro anteromolares; NMI: 1

NMI TOTAL: 6

\section{Comentarios:}

El material atribuible al género Talpa es muy escaso y fragmentario en El Sidrón: se trata de unas pocas piezas esqueléticas y fragmentos mandibulares que no presentan características diagnósticas suficientes para su determinación específica. Contrasta esta escasez con la abundancia de restos de Talpa europea que se documenta en otros yacimientos de edades similares como los niveles musterienses de El Castillo, en algunos de los cuales llega a representar entre la tercera parte del material hasta algo más de la mitad del porcentaje del NMI total de micromamíferos (Sesé, 2017a).

Actualmente en la península ibérica se encuentran las siguientes especies: Talpa europaea LINNAEUS, 1758 se distribuye en el tercio norte peninsular desde Prepirineos, Pirineos, montañas vascas y burgalesas y cordillera Cantábrica hasta el oriente asturiano, y en casi toda la Europa continental excepto Escandinavia y penínsulas mediterráneas hasta Siberia occidental. Talpa occidentalis CABRERA, 1907 es una especie endémica de la península ibérica que solo se encuentra en su tercio noroccidental, ausente en los Pirineos y la cuenca del Ebro. Ambas especies están completamente adaptadas a la vida subterránea; requieren suelos profundos de tierra húmeda fácilmente excavables y ricos en presas de invertebrados, aunque también se encuentran en prados y bosques con suelos bien formados, desde el nivel del mar hasta los $2.000 \mathrm{~m}$ de altitud. Recientemente se ha descrito una nueva especie, Talpa aquitania Nicolas, Martínez-Vargas \& Hugot, 2016, que se encuentra desde el norte de la península ibérica hasta el suroeste del Loira en Francia (Nicolas et al., 2016).

Orden Chiroptera BLUMENBACH, 1779

Suborden Microchiroptera DOBSON, 1875

Familia Rhinolophidae GRAY, 1866

Rhinolophus euryale BLASIUS, 1853 -

Rhinolophus mehelyi MATSCHIE, 1901

(Fig. 3: d)
Material por niveles y medidas:

- Zona E10, Nivel III: 1 fr. man. izq. con p4-m1$\mathrm{m} 2-\mathrm{m} 3$ que conserva la parte anterior con los alveolos de las unicúspides anteriores y la zona sinfisaria; NMI: 1

NMI TOTAL: 1

Medidas: $\mathrm{p} 4: \mathrm{L}=0,90 \mathrm{~mm} ; \mathrm{A}=0,79 \mathrm{~mm} ; \mathrm{m} 1$ : $\mathrm{L}=1,64 \mathrm{~mm} ; \mathrm{A}=0,95 \mathrm{~mm} ; \mathrm{m} 2 ; \mathrm{L}=1,54 \mathrm{~mm}$; $A=0,99 \mathrm{~mm} ; \mathrm{m} 3: \mathrm{L}=1,44 \mathrm{~mm} ; \mathrm{A}=0,94 \mathrm{~mm}$.

\section{Comentarios:}

La morfología de los dientes y de la mandíbula, con la curvatura de su parte anterior hacia el lado lingual, son característicos de los quirópteros. Su morfología y talla es similar a la de las especies de talla mediana del género Rhinolophus: Rhinolophus euryale y Rhinolophus mehelyi. Las medidas de dientes actuales de estas dos especies proporcionadas por Sevilla (1988) se solapan ampliamente, y asimismo las de los dientes de El Sidrón encajan en ambas, por lo que un material tan escaso no se puede atribuir a una u otra de estas especies con las que presenta similitud.

Actualmente $R$. euryale se distribuye por la península ibérica, sur de Europa, noroeste de África, Oriente Próximo, Turkmenistán e Irán. $R$. mehelyi tiene una distribución circunmediterránea, al sur del Sistema Central en la península ibérica, norte de África y sur de Europa hasta el Oriente Próximo Oriente e Irán. Según Palomo \& Gisbert (2002) las citas antiguas de esta última especie en algunas zonas del tercio norte peninsular podrían tratarse de $R$. euryale. Es la primera cita de una u otra de estas dos especies en un yacimiento del Pleistoceno Superior de la región cantábrica. Actualmente en el Principado de Asturias solo se encuentra $R$. euryale. Ambas especies son cavernícolas y termófilas.

Orden Rodentia BOWDICH, 1821

Suborden Sciuromorpha BRANDT, 1855

Familia Sciuridae FISCHER, 1817

\section{Marmota cf. marmota}

Material por niveles:

- Zona E23, Nivel II: 1 fr. de I inf. izq. que conserva gran parte de la zona apical; NMI: 1

NMI TOTAL: 1 
Además de este material se encontró, sin contexto estratigráfico seguro, 1 fr. de I sup. der. que conserva la parte apical.

\section{Comentarios:}

En una comparación del material de El Sidrón con el del género Marmota de las colecciones del MNCN, aquél presenta la misma talla y morfología. El material es tan escaso y fragmentario que realizamos con reservas su atribución a la especie Marmota marmota que es la que se registra durante el Pleistoceno en la península ibérica.

Marmota marmota se cita en numerosos yacimientos de la mitad norte de la península ibérica desde el Pleistoceno Inferior, Medio y Superior, especialmente en este último periodo, extinguiéndose al final de dicho periodo y comienzo del Holoceno (Sesé, 1994 y 2005; Arribas, 2004; López-García, 2008; García-Ibaibarriaga， 2015; Laplana et al., 2016). En la Europa continental, $M$. marmota se registra en numerosos yacimientos del Pleistoceno Medio y Superior (Kowalski, 2001), mientras que actualmente su distribución se ha reducido notablemente a poblaciones aisladas en algunos de los principales sistemas montañosos (Mitchell-Jones et al., 1999).

La marmota fue reintroducida a partir de mediados del siglo pasado en los Pirineos, primero en Francia y luego en España, donde ha prosperado con éxito. En los Pirineos actualmente suele vivir en zonas abiertas supraforestales subalpinas o alpinas, con suelos profundos fácilmente excavables y bastante cobertura herbácea, con o sin rocas, con preferencia por laderas soleadas de orientación sur en la vertiente pirenaica española, en la que su situación meridional atempera el clima riguroso de la montaña, en altitudes que varían entre los $1.300 \mathrm{~m}$ y los $2.900 \mathrm{~m}$ (Blanco, 1998a,b; Palomo \& Gisbert 2002). Los yacimientos pleistocenos de la península ibérica en los que se cita la presencia de M. marmota están situados generalmente a una altura moderada o baja, lo que hace suponer que durante el Pleistoceno no se trataba de una especie montana sino ligada a un hábitat de herbazales o estepa, tipo de hábitat que dicha especie encuentra actualmente en las zonas herbáceas abiertas que abundan por encima del área forestal pirenaica (Arribas, 2004).
Familia Gliridae MUIRHEAD, 1819

Eliomys quercinus (LINNAEUS, 1766)

\section{Material por niveles:}

- Zona F21, Nivel III: 1 fr. man. izq. con I y alveolos de premolar y molares; NMI: 1

- Zona N28/M28, Nivel I: 1 fr. max. sup. der. con P4-M1-M2-M3 y parte del arco zigomático; NMI: 1

NMI TOTAL: 2

\section{Comentarios:}

E1 P4 es de forma subtriangular. En los molares superiores, de superficie oclusal cóncava, además de las crestas principales existen dos centrolofos soldados a las cúspides labiales y entre sí, igual de largos en el primer y tercer molar y soldados entre sí, y más largo el anterior que el posterior en el segundo molar. Los molares presentan mucho desgaste de uso. En una comparación con material actual de Eliomys quercinus del MNCN el material de El Sidrón presenta la misma morfología y talla.

Esta especie estuvo muy extendida en la península ibérica durante el Pleistoceno Medio y Superior, pero fue infrecuente en la región cantábrica durante el Pleistoceno Superior, donde de hecho está ausente en numerosos yacimientos de dicha edad (Sesé, 2005), aunque recientemente se ha encontrado en algunos yacimientos como el Esquilleu (Sesé, 2005; Baena et al., 2005), Las Caldas (Laplana et al., 2006 y 2017), El Mirón (Cuenca-Bescós et al., 2008), Ventalaperra (Murelaga et al., 2007), Santimamiñe (Rofes et al., 2014), Antoliñako Koba (Zubeldia et al., 2007), Arlanpe (García-Ibaibarriaga et al., 2013) y Lezetxiki II (García-Ibaibarriaga et al., 2015).

Actualmente está ampliamente distribuida en la península ibérica y en el oeste y sur de Europa con escasas poblaciones en el norte y este de Europa, cuyo límite son los Urales y Dalmacia en la península balcánica, y en el norte de África. Aunque no es una especie estrictamente forestal se encuentra principalmente en bosques, pero también es frecuente en zonas abiertas arbustivas y rocosas como peñascales y pedrizas, desde el nivel del mar hasta más de 2.000 m de altura. Según Chaline (1972) la presencia de esta especie en el Pleistoceno se asocia con un cierto desarrollo forestal en un clima templado. 
Glis glis LINNAEUS, 1756

(Fig. 4: $a-b)$

Material por niveles:

- Zona F9/G5, Nivel III: 1 fr. man. der. con m1m2; 1 fr. man. der. con I, alveolos, premolar
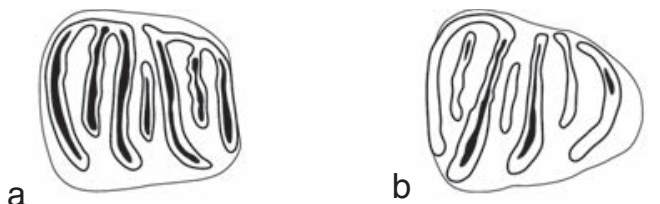

a
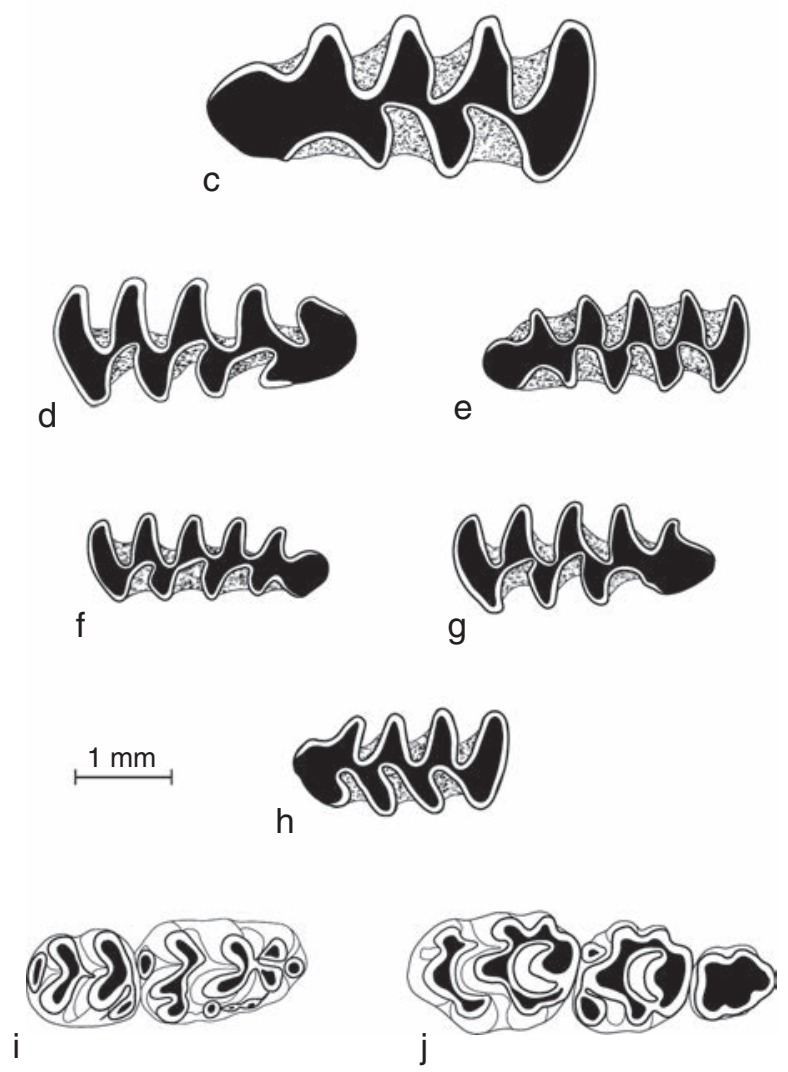

Fig. 4.-Dibujos de material dentario del yacimiento de la cueva de El Sidrón: Glis glis: a) m1 der. (Sidrón 2005; F9; 7; 9a III, 64, Caja 27); b): m3 izq. (Sidrón 2001; E23; 6; 2a , II, 56; Caja 4); Arvicola terrestris: c): m1 izq. (Sidrón 2000; H8; 1; 2a; 1221; Caja 7); Chionomys nivalis: d): m1 der. (Sidrón 2013; P-29/O29; I; Galería Osario ATC; Caja 88); Microtus arvalis - Microtus agrestis: e) m1 izq. (Sidrón 2002; G8; 7, 8, 9; III; 1145; Caja 14); Microtus lusitanicus: f) m1 der. (Sidrón 2006; G10; 5; 4a; 13; Caja 41); Microtus oeconomus: g) m1 der. (Sidrón 2013; P-29/O29; I; Galería Osario ATC; Caja 88); Clethrionomys glareolus: h) m1 izq. (Sidrón 2013; P-29/O29; Arreglo Corte; Caja 87); Apodemus sylvaticus Apodemus flavicollis: i) Serie dentaria der. m1-m2 (Sidrón 2003; G8; 8; 5a; III; 157; Caja 38); j) Serie dentaria izq. M1-M2-M3 (Sidrón 2006; F10; 9; 5a; 46; Caja 45). y molares y fr. de rama ascendente; 1 fr. man. der. con I y alveolos premolar y molares; $1 \mathrm{~m} 1$ der.; 1 fr. man. izq. con I, alveolos de premolar y molares, gran parte de rama ascendente y proceso articular completo; 1 fr. max. sup. izq. con alveolos de premolar y molares y arranque de arco zigomático; NMI: 3

- Zona E23, Nivel II: 1 m3 izq.; NMI: 1

NMI TOTAL: 4

Además de este material se encontraron, sin contexto estratigráfico seguro, $1 \mathrm{~m} 1$ izq. y $1 \mathrm{~m} 2$ der.

\section{Comentarios:}

Los dientes inferiores tienen la morfología y la talla característicos de Glis glis: superficie oclusal casi plana, $\mathrm{m} 1$ y $\mathrm{m} 2$ de contorno de rectangular a cuadrado y $\mathrm{m} 3$ de contorno subtriangular, cuatro crestas principales transversales al eje longitudinal del diente que suelen estar conectadas labialmente, que delimitan unos pequeños valles en el centro de los cuales se encuentran las crestas secundarias transversales que están aisladas y son más cortas (Chaline et al., 1974).

Como puede observarse en el apartado anterior, casi todo el material de este glírido apareció en una misma zona, y en esta zona la mayor parte de él en una misma muestra de una sola cuadrícula (G5).

Durante el Pleistoceno Superior y Holoceno Glis glis solo se registra en yacimientos del norte de la península ibérica (Sesé, 1994 y 2005; Arribas, 2004; López-García, 2008; García-Ibaibarriaga, 2015). Asimismo en la actualidad solo vive en el norte de la península ibérica y en toda Europa excepto la mayor parte de Escandinavia hasta el Cáucaso e Irán. Es una especie forestal y termófila (Pemán, 1985; Sesé, 2005). Actualmente habita en bosques generalmente caducifolios aunque también se encuentra en pinares en la Cataluña mediterránea y en bosques de coníferas en los Pirineos, hasta los $2.000 \mathrm{~m}$ de altitud (Blanco, 1998a,b; Palomo \& Gisbert, 2002).

Suborden Myomorpha BRANDT, 1855

Superfamilia Muroidea ILLIGER, 1811

Familia Cricetidae FISCHER, 1817

Subfamilia Arvicolinae GRAY, 1821

Arvicola terrestris (LINNAEUS, 1758)

(Fig. 4: c) 
Material por niveles:

A continuación, se da la relación del material craneal. En primer lugar el material dentario que está en mandíbulas y en el cuadro final el número de $\mathrm{m} 1$ der. e izq. en el que se basa el NMI.

- Zona E/F/G/H/4-10, Nivel III: 14 fr. man. con dientes; 1 fr. cráneo con prácticamente toda la bóveda craneal, parte de los arcos zigomáticos, serie dentaria der. con I-M1-M2-M3 y serie dentaria izq. con I-M1-M2-M3; 1 fr. cráneo con max. der. con I y alveolos de primeros molares y max. izq. con alveolos primer molar y M2.

- Zona H8, Nivel IV: 1 man. izq. casi completa con I-m1-m2-m3.

- Zona F/21-22-23, Nivel III: 4 fr. man. con dientes; 2 fr. man. sin dientes.

- Zona I/J/24-25, Nivel I: 1 fr. man. izq. con I-m1-m2; $1 \mathrm{fr}$. cráneo con bóveda craneal son series dentarias I-M1-M2-M3 der. e I-M1-M2-M3 izq.

- Zona I/J/24-25, Nivel II: 2 m/M (no m1)

- Zona K/L/M/N/P/O/27-30, Nivel I: 7 fr. mand. con dientes (3 de ellas con rama ascendente casi completa)

- Zona K/O/P/26-29, Nivel I/III: 1 fr. cráneo con max. der. con I-M1-M2 y max. izq. con I-M1-M2

Número de $M_{1}$ y NMI por zonas y niveles:

\begin{tabular}{lcccccc}
\hline Zona & Nivel & $\begin{array}{c}\mathrm{N}^{\circ} \mathrm{m} 1 \\
\text { der. }\end{array}$ & $\begin{array}{c}\mathrm{N}^{\circ} \mathrm{m} 1 \\
\text { izq. }\end{array}$ & $\begin{array}{c}\text { Otro } \\
\text { tipo de } \\
\text { molar }\end{array}$ & $\begin{array}{c}\mathrm{N}^{\circ} \mathrm{m} 1 \\
\text { Totales }\end{array}$ & $\mathrm{NMI}$ \\
\hline E/F/G/H/4-10 & III & 8 & 9 & & 17 & 9 \\
H8 & IV & - & 2 & & 2 & 2 \\
F21 & I & 1 & - & & 1 & 1 \\
F/20-21-22 & III & 1 & 4 & & 5 & 4 \\
E23-24 & II & 2 & 1 & & 3 & 2 \\
I/J/24-25 & I & - & 1 & & 1 & 1 \\
I/J/24-25 & II & - & - & $(2)$ & - & $(1)$ \\
K/L/M/N/ & I & 4 & 5 & & 9 & 5 \\
O/P/27-30 & & & & & & 1 \\
M/N/O/27-29 & III & 1 & - & & 1 & 1 \\
K/O/P/26-29 & I-III & - & - & $(4)$ & & $(1)$ \\
TOTAL & TOTAL & 17 & 22 & & 39 & 27 \\
\hline & & & & & & \\
\hline
\end{tabular}

Además de este material se encontraron, sin contexto estratigráfico seguro: 4 fr. man. der. con la serie dentaria completa $\mathrm{m} 1-\mathrm{m} 2-\mathrm{m} 3 ; 2$ fr. man. der. con algunos molares; 5 fr. man. izq. con la serie dentaria completa $\mathrm{m} 1-\mathrm{m} 2-\mathrm{m} 3 ; 4$ fr. man. izq. con algunos molares; 6 fr. craneales con algunos molares; 2 fr. max. sup. con algunos molares.

\section{Comentarios:}

Los molares son arrizodontos y presentan cemento en los ángulos entrantes. Los $\mathrm{m} 1$ de El Sidrón presentan el esmalte más grueso en la parte mesial de los triángulos que en la distal, tal como sucede en Arvicola terrestris (denominada también por algunos autores Arvicola amphibius LINNAEUS, 1758), a diferencia de Arvicola sapidus MILLER, 1908 en los que el esmalte es más grueso en la parte distal de los triángulos que en la mesial.

En el norte de la península ibérica $A$. terrestris se registra en numerosos yacimientos del Pleistoceno Superior (Sesé, 1994 y 2005; Sesé \& Sevilla, 1996; Arribas, 2004; López-García, 2008; Bañuls et al., 2012; Laplana et al., 2013 y 2016). Respecto a la cita de esta especie en un yacimiento peninsular del Pleistoceno Superior tan meridional como la Cueva de Ambrosio (Almería) (Sesé \& Soto, 1988), según la revisión realizada por Sesé (2017a) sobre el material de dicho yacimiento, se trata de la especie $A$. sapidus.

Actualmente vive en el norte de la península ibérica, en casi toda Europa y en gran parte de Asia hasta el océano Glacial Ártico y cerca del Pacífico. En la península ibérica es una especie hipogea que construye madrigueras en suelos profundos, blandos y húmedos, generalmente en prados naturales, en altitudes que varían desde pocos metros por encima del nivel del mar hasta los 2.000 metros de altitud.

\section{Chionomys nivalis (MARTINS, 1842)}

(Fig. 4: d)

Material por niveles:

- Zona O/P/Q/29-30, Nivel I: 1 m1 der.; NMI: 1 NMI TOTAL: 1

\section{Comentarios:}

Este diente presenta la siguiente morfología: arrizodonto, con cemento en los ángulos entrantes, esmalte más grueso que en el género Microtus; en superficie oclusal consta de un lóbulo posterior, cuatro triángulos cerrados, el T5 es confluyente por un cuello estrecho con la cúspide anterior que tiene una forma lanceolada o subtriangular, en la que los 
triángulos T6 y T7 están menos desarrollados que los demás triángulos; esta morfología del complejo anterocónido corresponde al morfotipo "nivaloide" característico de Chionomys nivalis (Chaline, 1972).

Esta especie se registra en numerosos yacimientos del Pleistoceno Superior y Holoceno de la mitad norte y sureste de la península ibérica, generalmente situados en sistemas montañosos (Sesé 1994 y 2005; Sesé \& Sevilla, 1996; Arribas, 2004; López-García, 2008; García-Ibaibarriaga, 2015). Asimismo, durante el Pleistoceno Superior y Holoceno se encuentra en muchos yacimientos de toda Europa situados en zonas montañosas (Kowalski, 2001). En la actualidad solo se encuentra en los principales sistemas montañosos de la España peninsular: en los Pirineos, cornisa cantábrica, montañas gallegas, Sierra Cebollera, sierras de los Ancares, de la Demanda, Gredos, Guadarrama y Sierra Nevada (Palomo \& Gisbert, 2002); y en los principales sistemas montañosos euroasiáticos: Alpes, Apeninos, Polonia, Montes Tatra, Rumania, Cárpatos, Balcanes, Grecia, Turquía, Israel, Líbano, Siria, Irán, Ucrania, Rusia y Cáucaso (Mitchell-Jones et al., 1999; Palomo \& Gisbert, 2002; Wilson \& Reeder, 2005).

Chionomys nivalis es una especie rupícola y heliófila, bien adaptada a los ambientes de alta montaña, que vive en laderas generalmente orientadas al sur, en altitudes generalmente entre los 1.000 y los 4.700 $\mathrm{m}$ de altitud, aunque en algunas áreas se encuentra en altitudes mucho menores, en áreas rocosas con pedreras estables y escasa cobertura vegetal, y más raramente en zonas arbustivas, herbáceas o de claros de bosques (Mitchell-Jones et al., 1999; Blanco, 1998a,b; Palomo \& Gisbert, 2002). Está más asociada a medios abiertos pedregosos que a la alta montaña, según Kowalski (1992), ya que en algunas zonas montañosas desciende hasta casi la altura del nivel del mar.

\section{Microtus arvalis (PALLAS, 1778) - Microtus agrestis (LINNAEUS, 1761)}

(Fig. 4: e)

\section{Material por niveles:}

A continuación se da la relación del material craneal. En primer lugar el material dentario que está en mandíbulas y en el cuadro final el número de $\mathrm{m} 1$ der. e izq. en el que se basa el NMI.
- Zona E/F/G/H/4-10, Nivel III: 1 man. der. con $\mathrm{m} 1-\mathrm{m} 2-\mathrm{m} 3 ; 1$ fr. man. der. con m1; 1 fr. man izq. con $\mathrm{m} 1-\mathrm{m} 2$.

- Zona $\mathrm{I} / \mathrm{J} / 24-25$, Nivel I: 1 fr. man. der. con $\mathrm{m} 1-\mathrm{m} 2-\mathrm{m} 3 ; 1$ fr. man. izq. con I-m1-m2-m3; 1 fr. parte superior craneal con I-M2-M3 der. e I-M1-M2 izq.

- Zona N/MO/P/Q/28-29-30, Nivel I: 1 fr. man. der. con m1-m2.

\begin{tabular}{lccccc} 
Número de $M_{1} y$ & NMI por zonas & niveles: \\
\hline Zona & Nivel & $\begin{array}{c}\mathrm{N}^{\circ} \mathrm{m} 1 \\
\text { der. }\end{array}$ & $\begin{array}{c}\mathrm{N}^{\circ} \mathrm{m} 1 \\
\text { izq. }\end{array}$ & $\begin{array}{c}\mathrm{N}^{\circ} \mathrm{m} 1 \\
\text { Totales }\end{array}$ & $\mathrm{NMI}$ \\
\hline E/F/G/H/4-10 & III & 3 & 1 & 4 & 3 \\
F20-21-22 & III & 2 & - & 2 & 2 \\
E23-24 & II & - & 1 & 1 & 1 \\
I/J/24-25 & I & 1 & 1 & 2 & 1 \\
I/J/24-25 & II & - & 1 & 1 & 1 \\
N/M/O/P/Q/28-29-30 & I & 1 & 1 & 2 & 1 \\
TOTAL & TOTAL & 7 & 5 & 12 & 9 \\
\hline
\end{tabular}

Además de este material se encontraron, sin contexto estratigráfico seguro: 2 fr. man. der. con I-m1m2-m3; 1 fr. man. izq. con I-m1-m2-m3; 1 fr. cráneo con bóveda craneal y arranque de los arcos zigomáticos, I-M1-M2-M3 der. e I-M1-M2-M3 izq.

\section{Comentarios:}

La morfología de los $\mathrm{m} 1$ es la siguiente: arrizodontos, con cemento en los ángulos, entrantes; en superficie oclusal presentan un lóbulo posterior, cinco triángulos cerrados y un complejo anterior que, teniendo en cuenta los morfotipos descritos por Chaline (1972), varía de la forma siguiente en los $12 \mathrm{~m} 1$ de El Sidrón en los que se aprecia claramente la morfología: 8 ejemplares presentan el morfotipo de tipo "arvalis" característico de $M$. arvalis, con los triángulos T6 y T7 mas o menos opuestos entre sí y confluyentes en la cúspide anterior que es de contorno ovoide o redondeado; 2 especímenes corresponden al morfotipo "agrestis", característico de $M$. agrestis, en el que los triángulos T6 y T7 son alternos y confluyen en las cúspide anterior que es de contorno ovoide, con el T6 casi cerrado y aislado del T7 que se abre a la cúspide anterior; y finalmente 2 ejemplares presentan una morfología intermedia entre ambos morfotipos; 
razón por la que damos una denominación taxonómica en conjunto de ambas especies, tal como se encuentra en muchos yacimientos del norte de la península ibérica (Sesé, 2005), indicando que están las dos especies en el yacimiento de El Sidrón, siendo $M$. arvalis la más abundante como se ha indicado antes.

$M$. arvalis y $M$. agrestis se registran en la península ibérica desde la última parte del Pleistoceno Medio (Sesé \& Gil, 1988) y en muchos yacimientos del Pleistoceno Superior y Holoceno hasta la actualidad de la mitad norte peninsular (Sesé, 1994 y 2005; Arribas, 2004; López-García, 2008; GarcíaIbaibarriaga, 2015).

$M$. arvalis actualmente se encuentra ampliamente distribuida por la Europa continental, excepto en Escandinavia y las islas y regiones mediterráneas como el sur la península ibérica, de Italia, y Grecia, hasta el centro de Rusia, (Mitchell-Jones et al., 1999). Vive en espacios abiertos con abundante cobertura vegetal, arbustiva o herbácea, desde los 500 a los 2.000 metros de altitud (Palomo \& Gisbert, 2002).

M. agrestis está ampliamente distribuida actualmente por casi toda Eurasia hasta Siberia y noroeste de China, excepto en el sur de la península ibérica, Irlanda, islas mediterráneas, Italia y Grecia y (Mitchell-Jones et al., 1999). Se encuentra en zonas húmedas, preferentemente en herbazales y praderas, y también en bosques con suelo herbáceo desarrollado, en márgenes de arroyos y zonas húmedas de prados y juncales, desde el nivel del mar hasta una altitud por encima de los $1.600 \mathrm{~m}$ (Palomo \& Gisbert, 2002).

\section{Microtus lusitanicus (GERBE, 1879)}

(Fig. 4: f)

Material por niveles:

A continuación, se da la relación del material craneal. En primer lugar el material dentario que está en mandíbulas y en el cuadro final el número de $\mathrm{m} 1$ der. e izq. en el que se basa el NMI.

- Zona F/G/H/8-10, Nivel III: 1 fr. man. der. con I-m1-m2-m3; 1 fr. man. der con m1; 1 fr. man izq. con I-m1-m2-m3, 1 fr. man izq. con I-m1-m2.

- Zona M/28-29, Nivel III: 1 fr. man izq. con m1.
Número de $M_{1} y$ NMI por zonas y niveles:

\begin{tabular}{lccccc}
\hline Zona & Nivel & $\begin{array}{c}\mathrm{N}^{\circ} \mathrm{m} 1 \\
\text { der. }\end{array}$ & $\begin{array}{c}\mathrm{N}^{\circ} \mathrm{m} 1 \\
\text { izq. }\end{array}$ & $\begin{array}{c}\mathrm{N}^{\circ} \mathrm{m} 1 \\
\text { Totales }\end{array}$ & $\mathrm{NMI}$ \\
\hline F/G/H/8-10 & III & 3 & 2 & 5 & 3 \\
F20-21-22 & III & 2 & 2 & 4 & 2 \\
E23 & II & 1 & - & 1 & 1 \\
M/28-29 & III & 1 & - & 1 & 1 \\
TOTAL & TOTAL & 7 & 4 & 11 & 7 \\
\hline
\end{tabular}

Además de este material se encontraron, sin contexto estratigráfico seguro: 2 fr. man. der. con I-m1$\mathrm{m} 2-\mathrm{m} 3 ; 2$ fr. man. der. con I-m1-m2; 1 fr. man. izq. con I-m1-m2-m3; 1 m1 izq.

\section{Comentarios:}

Los $\mathrm{m} 1$ presentan la siguiente morfología: arrizodontos, con cemento en los ángulos, entrantes; en vista oclusal presentan: un lóbulo posterior; tres primeros triángulos cerrados; triángulos T4 y T5 confluyentes y enfrentados formando un "rombo pitimiano"; triángulos T6 y T7 formando un segundo rombo pitimiano, ambos bien desarrollados prácticamente como los demás triángulos, enfrentados y confluyendo por un cuello relativamente estrecho en la cúspide anterior que es redondeada, ovalada o alargada; esta morfología del complejo anterocónido es la característica de Microtus lusitanicus según Chaline (1972).

Esta especie se registra en el Pleistoceno Superior en muchos yacimientos de la mitad norte peninsular, especialmente en la región cantábrica, y en algunos yacimientos de los Pirineos franceses (Sesé, 2005; Arribas, 2004; LópezGarcía, 2008).

M. lusitanicus es una especie endémica de la península ibérica que actualmente solo vive en el noroeste de la península ibérica y suroeste de Francia. Está ligada a suelos blandos y húmedos con una densa cobertura vegetal, generalmente en praderas, aunque también se encuentra en zonas boscosas y a orillas de los ríos, desde el nivel del mar hasta los 2.000 m de altitud (Mitchell-Jones et al., 1999; Palomo \& Gisbert, 2002).

Microtus oeconomus (PALLAS, 1776)

(Fig. 4: g) 
Material por niveles:

- Zona O/P/Q/29-30, Nivel I: 2 fr. man. der. con I-m1-m2-m3; 2 fr. man der. con I m1-m2; 1 m1 der.; 1 fr. man. izq. con m1-m2-m3; NMI = 4 NMI TOTAL: 1

Además de este material se encontró, sin contexto estratigráfico seguro: 1 fr. man. der. con I-m1-m2.

\section{Comentarios:}

La morfología de los $\mathrm{m} 1$ es la siguiente: arrizodontos; con cemento en los ángulos entrantes; en vista oclusal consta de: lóbulo posterior; cuatro triángulos cerrados; T5 ampliamente confluyente por un cuello amplio y alargado con la cúspide anterior, en la que también confluye el incipiente T7; no hay T6; esta morfología del complejo anterocónido corresponde al morfotipo "raticepoide" característico de Microtus oeconomus (= Microtus ratticeps (KEYSERLING \& BLASIUS, 1841) (Chaline, 1972).

Aunque el material es escaso cabe señalar que el que está en contexto estratigráfico se concentra en una sola zona y nivel con un NMI de 4 .

Esta especie durante el Pleistoceno Superior se registra en muchos yacimientos de la mitad norte de la península ibérica, la mayoría de ellos en la región cantábrica, (Sesé, 1994 y 2005; Sesé \& Sevilla, 1996; Arribas, 2004; López-García, 2008; García-Ibaibarriaga, 2015), algunos en los Pirineos orientales (López-García, 2008), y unos pocos en el Sistema Central que constituyen su registro más meridional en Europa (Sevilla et al., 2011). Persistió en la península ibérica en el Holoceno, aunque con escasos hallazgos, hasta su extinción en tiempos históricos, siendo su último registro en un nivel tardorromano del yacimiento de Amalda (Pemán, 1990).

Actualmente $M$. oeconomus vive en regiones septentrionales euroasiáticas, aunque no de forma continua, desde Europa central hasta el mar de Bering y en Alaska en norte América (Mitchell-Jones et al., 1999; Wilson \& Reeder, 2005). Durante el Pleistoceno Superior, sin embargo, tuvo una distribución euroasiática mucho más amplia en regiones y países en los que no existe actualmente como España, Francia, Italia, Alemania y Gran Bretaña (Kowalski, 2001), por lo que su registro fósil documenta una reducción en su distribución geográfica actual respecto a dicho periodo. $M$. oeconomus vive preferentemente en zonas con gran cobertura vegetal húmeda, en suelos saturados como prados húmedos, turberas, pantanos, y orillas de arroyos, ríos, lagos y playas, aunque en invierno emigran a zonas más secas de praderas herbáceas (Mitchell-Jones et al., 1999).

\section{Clethrionomys glareolus (SCHREBER, 1780)}

(Fig. 4: h)

\section{Material:}

Sin contexto estratigráfico seguro se ha encontrado 1 fr. man. izq. con I-m1-m2-m3; NMI: 1

NMI TOTAL: 1

\section{Comentarios:}

Los molares son rizodontos, con cemento en los ángulos entrantes y esmalte grueso. El $\mathrm{m} 1$ presenta la siguiente morfología en superficie oclusal: un lóbulo posterior, triángulos $\mathrm{T} 1, \mathrm{~T} 2$ y T3 con cierta comunicación entre ellos respectivamente; el T4 está separado del T5 y de la cúspide anterior por un cuello estrecho; el T5 confluye en la cúspide anterior por un cuello amplio; hay un T6 esbozado; la cúspide anterior tiene una forma lanceolada; esta morfología es la característica de Clethrionomys glareolus.

En la península ibérica esta especie se registra en algunos yacimientos del Pleistoceno Superior de la mitad norte (Sesé, 2005; Alcaraz et al., 2017; Laplana et al., 2013, 2016) y del sur (Delgado Castilla et al., 1993). Actualmente en la península ibérica solo se encuentra en el tercio norte, aunque está ampliamente distribuida en el resto de Europa y Asia hasta el Lago Baikal y norte de Asia Menor. Es una especie típicamente forestal que se encuentra desde el nivel del mar hasta los $2.400 \mathrm{~m}$ de altitud (Palomo \& Gisbert, 2002; Mitchell-Jones et al., 1999).

Familia Muridae ILLIGER, 1811

Subfamilia Murinae ILLIGER, 1811

Apodemus sylvaticus (LINNAEUS, 1758) Apodemus flavicollis (MELCHIOR, 1834)

(Fig. 4: i-j)

\section{Material por niveles:}

- Zona F/G/H/8-10, Nivel III: 1 fr. man der. con I-m1-m2-m3; 1 fr. man. der. con I-m1-m2; 1 fr. man. der. sin dientes con alveolos de los molares; 3 fr. man. izq. con alveolos de los molares; NMI: 3 
- Zona F20, Nivel III: 1 fr. man. izq. con I-m1-m2; NMI: 1

- Zona E23, Nivel II: 1 M2; NMI: 1

- Zonas $\mathrm{O} / \mathrm{P} / \mathrm{Q} / 29-30$, Nivel I: 1 fr. man. der. con I-m1-m2; NMI; 1

NMI TOTAL: 6

Además de este material se encontraron, sin contexto estratigráfico seguro: 1 fr. man. der. con m1-m2; 1 fr. man. der. sin dientes con alveolos de molares; 1 fr. man. izq. con $\mathrm{m} 2 ; 1$ fr. man izq. sin dientes con alveolos de molares; 1 fr. craneal en varios fragmentos: max. sup. con M1-M2-M3 der. y M1-M2-M3 izq. con parte anterior craneal y parte de los arcos zigomáticos, man. der. con I-m1-m2-m3 y man. izq. con I-m1-m2-m3 y 4 fr. craneales; 1 fr. max. sup. izq. con M1-M2-M3.

\section{Comentarios:}

El material es muy escaso y no permite atribuirlo a ninguna de las dos especies a las que es semejante en talla y morfología: Apodemus sylvaticus y Apodemus flavicollis, especies que es posible distinguir fundamentalmente cuando se dispone de muchos m2/M2, según Michaux \& Pasquier (1974), lo que no es el caso del yacimiento de El Sidrón, por lo que realizamos su denominación conjunta como Apodemus sylvaticus - Apodemus flavicollis.

En Europa el registro de las dos especies empieza en el Villafranquiense según Kowalski (2001). Ambas están en muchos yacimientos del Pleistoceno y Holoceno de la península ibérica (Sesé, 1994 y 2005; Sesé \& Sevilla, 1996; Arribas, 2004; LópezGarcía, 2008; García-Ibaibarriaga, 2015).

A. sylvaticus se distribuye ampliamente en la actualidad en Europa desde la península ibérica (donde es uno de los micromamíferos mas abundantes y extendidos) hasta el sur de Escandinavia, oeste de Rusia, Asia Menor, norte de África, islas mediterráneas, Irlanda, Gran Bretaña e Islandia (MitchellJones et al., 1999; Palomo \& Gisbert, 2002). Vive preferentemente en zonas con una buena cobertura vegetal arbórea o arbustiva, aunque se encuentra en una gran variedad de hábitats, incluso en condiciones de clima mediterráneo áridas (Mitchell-Jones et al., 1999; Palomo \& Gisbert, 2002).

A. flavicollis se distribuye actualmente desde el tercio norte de la península ibérica, por Europa, sur de Noruega, Suecia, Finlandia y Gran Bretaña, hasta los Urales, Cáucaso, los Balcanes y Palestina. Es una especie forestal que suele vivir en bosques húmedos de caducifolios o de galería de ríos y arroyos, aunque también se encuentra en áreas con una densa cobertura arbustiva o herbácea, pero generalmente arboladas (Blanco, 1998a,b; Palomo \& Gisbert, 2002).

Orden Lagomorpha BRANDT, 1855

Leporidae FISCHER, 1817

Oryctolagus cuniculus LINNAEUS, 1758

(Fig. 5: a)

\section{Material por niveles:}

- Zona F/G/7-9, Nivel III: 1 fr. p3 der.; 1 fr. I sup.; NMI: 1

- Zona H8, Nivel IV: 1 fr. man. izq. con p4m1-m2; NMI: 1

- Zona F21, Nivel I: 2 fr. p/P/-m/M; NMI: 1

- Zona F/20-21, Nivel III: 1 d3 der.; 1 p3 der.; 1 fr. p3 der.; 1 p3 izq.; 2 p/m; 2 fr. I inf.; 1 fr. man. der. sin dientes con alveolos premolares; $3 \mathrm{P} / \mathrm{M}$; 4 I sup.; NMI: 3

- Zona E24, Nivel II: 2 p3 der.; 1 p3 izq.; 5 fr. p/m; 1 P2 izq.; 4 P/M; NMI: 2

- Zona I/J24-25, Nivel II: 1 I inf.; 2 P/M; NMI: 1

- Zona O/P/Q/29-30, Nivel I: 1 fr. man. der. con d3-d4-m1-m2-m3; NMI: 1

- Zona M-27-28, Nivel III: 1 I inf.; 1 I sup.; NMI: 1 NMI TOTAL: 11

Además de este material se encontraron, sin contexto estratigráfico seguro: 1 man. izq. con d3-d4m1-m2-m3; 1 fr. man. izq. con I-p3-m1-m2-m3; 1 fr. cráneo con max. sup. der. con la serie dentaria: P2-P3-P4-M1-M2-M3 y max. sup. izq. sin dientes;

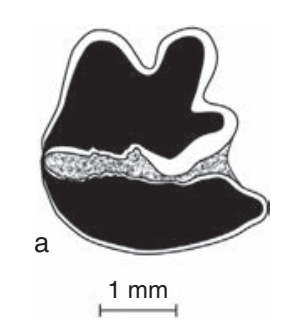

Fig. 5.-Dibujo de material dentario del yacimiento de la Cueva de El Sidrón: Oryctolagus cuniculus: a) p3 der. (Sidrón 2001; E23; 9; 6а; II; 68; Caja 6). 
1 fr. max. sup. der. con la serie dentaria: P2-P3-P4M1-M2-M3 y parte del arco zigomático; 1 I inf.; 1 fr. p3 izq.; 3 p/m; 2 P/M.

\section{Comentarios:}

El p3 presenta la morfología característica de Oryctolagus cuniculus que le distingue del género Lepus, según López Martínez (1989): anterocónidos subiguales, anterofléxido ancho y ortogonal, protofléxido ancho y poco profundo, hipofléxido poco rugoso y no estrangulado. Una vez determinada esta especie en el yacimiento, se ha adscrito el resto del material de lepóridos de las distintas zonas y niveles a la misma, aunque no presente características morfológicas con valor diagnóstico, porque su talla y morfología son acordes con dicha especie.

El conejo, y en general los lagomorfos, son muy escasos en los numerosos yacimientos del Pleistoceno Superior y Holoceno de la región cantábrica (Altuna, 1972; Sesé, 2005), y cuando se registran suelen estar representados por un escaso número de restos, a diferencia de lo que ocurre en muchos yacimientos del centro y de la mitad sur de la península ibérica, tanto del Pleistoceno Medio como del Pleistoceno Superior, en los que suelen ser muy abundantes (Sesé, 2005). Sin embargo, en el yacimiento asturiano de Coímbre, en niveles del Magdaleniense superior datados en 15.680-14.230 cal ka, es decir, de cronologías más recientes que las de de El Sidrón, excepcionalmente se registra una gran abundancia de restos de conejo (entre el 21\% y el 23\% del NMI en los niveles Co.B.1a y Co.B.1b), muy posiblemente debido en su mayor parte a la acción humana (Sesé, 2017b). En el yacimiento de El Sidrón, aunque Oryctolagus cuniculus es la segunda especie más abundante, está representada con un porcentaje del 13,3\% del total del NMI (véase la Tabla 1), porcentaje que es mucho menor que el de la especie más abundante que es Arvicola terrestris con el 32,5\% del total del NMI.

Según nuestras observaciones, dado que hay restos de conejo, aunque pocos, en prácticamente todos los sectores, zonas y niveles del yacimiento; que no se han observado restos en conexión anatómica; que no se han encontrado individuos juveniles, algo que podría suponer una mortandad selectiva; y que la coloración y morfología de los fósiles es como las de los demás restos fósiles de micromamíferos, descartamos que sean producto de una intrusión por la existencia de madrigueras en la cueva.

Oryctolagus cuniculus se originó en la península ibérica en el Pleistoceno Medio (López Martínez, 1989), desde donde se extendió al resto de Europa, y en tiempos históricos a otros continentes debido a la introducción humana (Mitchell-Jones et al., 1999).

El conejo está ampliamente distribuido actualmente en la península ibérica, aunque es muy escaso en el norte, especialmente en Asturias en donde está prácticamente ausente excepto en algunos lugares, y es más abundante en la región mediterránea y mitad sur peninsular. Las condiciones óptimas para esta especie son el clima árido y caluroso, con precipitaciones en torno a los $500 \mathrm{~mm}$ anuales, en áreas de matorral y dehesas, aunque a pequeña escala es abundante en las cercanías de zonas ribereñas, por debajo de los $1.000 \mathrm{~m}$, siendo raro a partir de los $1.500 \mathrm{~m}$ de altitud, (Blanco, 1998a,b; Palomo \& Gisbert, 2002).

\section{Análisis de los micromamíferos de El Sidrón}

\section{Consideraciones sobre la asociación de micromamíferos}

Casi todos estos taxones viven actualmente en la península ibérica y en Asturias donde se localiza el yacimiento excepto los siguientes: Microtus oeconomus que se extinguió en la península ibérica en épocas históricas y se distribuye en la actualidad ampliamente en Eurasia a partir de Europa central, aunque no de forma continua, y en Alaska. Marmota marmota también se extinguió en la península ibérica al final del Pleistoceno Superior o comienzos del Holoceno, aunque ha sido reintroducida con éxito en los Pirineos a mediados del siglo XX. Con respecto a Oryctolagus cuniculus, muy abundante en casi toda la península ibérica, cabe señalar que está prácticamente ausente de Asturias excepto en unos pocos enclaves puntuales.

La asociación de El Sidrón presenta una gran diversidad con hasta 16 taxones diferentes teniendo en cuenta que bajo la denominación de Microtus arvalis-Microtus agrestis están presentes las dos especies indicadas. A pesar de ello, hay bastantes taxones, como Rhinolophus euryale-Rhinolophus mehelyi, Marmota cf. marmota, Eliomys quercinus, 
Chionomys nivalis y Clethrionomys glareolus representadas tan solo por un individuo, o, como en el caso de Microtus oeconomus, con escasos individuos concentrados en un solo nivel y en una sola muestra. Es muy notable la ausencia en todos los niveles de El Sidrón de Pliomys lenki, una especie que es tan común en la mayoría de las asociaciones del Pleistoceno Superior de cronologías similares a las de este yacimiento, especialmente en la región cantábrica, hasta su extinción hacia el final de dicho periodo (Sesé, 2005). La explicación de su ausencia en El Sidrón no parece que sea la escasa penetración de la especie hacia la parte occidental de la región cantábrica, ya que, aunque escaso en número de restos, se registra en el yacimiento también asturiano de Las Caldas, aunque de edad más reciente que la de El Sidrón (Laplana et al., 2006 y 2017), por lo que podría deberse a cuestiones tafonómicas de falta de registro o bien quizás a causas medioambientales desfavorables para su presencia en los alrededores del yacimiento en el momento de su formación.

El yacimiento de El Sidrón no es rico en restos de micromamíferos (con un NMI total de 83; de 84 contabilizando Clethrionomys glareolus; véase la Tabla 1) en comparación con otros yacimientos de la región cantábrica en los que el NMI se cuenta en algunos casos por cientos o incluso miles, como el de la cueva de El Castillo en el que hay niveles de edades similares (Sesé, 2017a). La zona mejor representada en El Sidrón es la de las cuadrículas $\mathrm{E} / \mathrm{F} / \mathrm{G} / \mathrm{H} / 4-10$, nivel III, en la que aparecieron también los restos humanos, con 11 de los 16 taxones de micromamíferos registrados en el yacimiento y un NMI de 26. Las demás zonas y niveles están muy escasamente representadas en cuanto al NMI (entre un NMI de 16 y 4), por lo que son poco representativas, aunque en algunas de ellas se registran taxones que no aparecen en la zona mencionada anteriormente, como Marmota cf. marmota y Eliomys quercinus (que se encuentran en el sector 4), y Chionomys nivalis y Microtus oeconomus (que están en el sector 1) (Fig.1). Hay que tener en cuenta la diferente potencia estratigráfica de las distintas zonas y niveles, y el tipo de depósito y su procedencia (Rasilla et al., 2011 y 2014; Cañaveras et al., e.p), y que hay también bastantes restos de micromamíferos sin contexto estratigráfico seguro debido generalmente a las removilizaciones por las periódicas inundaciones de la galería (tal como se ha indicado en los apartados respectivos de varios de los taxones), que al no poder ser tenidos en cuenta en el recuento por zonas y niveles, empobrecen los resultados que sí son válidos. Es muy posible también que esta disparidad de representación en las diferentes zonas, en la que parece evidente que hay más restos de micromamíferos en la zona que es también muy rica en fósiles humanos, y menos restos de micromamíferos en las zonas alejadas de esta, esté relacionada con una posible mayor aportación de sedimentos por la cercanía a la comunicación con el exterior de la primera zona indicada, en el momento de formación de los depósitos.

Se pueden hacer algunas observaciones con respecto a la representación de los taxones por zonas y niveles (Tabla 1): Los taxones relativamente más abundantes en el conjunto de zonas y niveles del yacimiento: Arvicola terrestris (32,5\%), Oryctolagus cuniculus (13,3\%), Microtus arvalis-Microtus agrestis $(10,8 \%)$, Microtus lusitanicus $(8,4 \%)$, son también los que se registran prácticamente en casi todas las zonas; y en menor medida también presentan bastante continuidad, aunque no en todas las zonas y niveles: Apodemus sylvaticus - Apodemus flavicollis (7,2\%), Talpa sp. (7,2\%) y Sorex araneus - Sorex coronatus $(3,6 \%)$. Cabe señalar que Microtus oeconomus, con un $4,8 \%$ de los restos, solo se registra en una sola zona y un mismo nivel pero con un NMI de 4 , lo que parece una relativa buena concentración de este taxón en dicha muestra en relación a las demás zonas de las que está ausente. El resto de los taxones está representado por un NMI de 4 y un porcentaje del NMI de 4,8\% (Glis glis), un NMI de 2 y un porcentaje del NMI de 2,4\% (Eliomys quercinus), y un NMI de 1 y un porcentaje del NMI de 1,2\% (Neomys cf. fodiens, Rhinolophus euryale-Rhinolophus mehelyi, Marmota cf. marmota y Chionomys nivalis). Sin embargo, la asociación faunística de micromamíferos de El Sidrón, en su conjunto, es similar en su composición a las asociaciones que se encuentran en muchos de los yacimientos del Pleistoceno Superior de la región cantábrica (Sesé, 2005).

\section{Observaciones tafonómicas}

Sobre el material de micromamíferos hallado en El Sidrón se pueden hacer las siguientes consideraciones tafonómicas: hay una gran abundancia 
de restos esqueléticos especialmente craneales y dentarios; el material está en muy buen estado de conservación en general: hay numerosos cráneos y mandíbulas que conservan toda la dentición; en algunas muestras hay cráneos y mandíbulas de determinados taxones que podrían corresponder al mismo individuo; en muchas piezas craneales esta buena conservación está favorecida por la compactación del sedimento y concreciones calizas en torno a los fósiles que han propiciado su preservación frente a roturas recientes debidas a los procesos de extracción del material; aunque hay una gran diversidad de taxones, solo predominan unos pocos, mientras que el resto están escasamente representados; no se han encontrado restos esqueléticos en conexión anatómica; ninguno de los taxones habita en cuevas, excepto el quiróptero que está por otra parte representado por un solo ejemplar, y también es susceptible de ser cazado; finalmente, unos pocos dientes presentan los efectos de la digestión, aunque débil en todos los casos. Así, de un total de 142 molares, en 135 no hay ninguna huella de digestión y solo 7 (un 5,2\%) presentan un grado de digestión débil según los grados definidos por Andrews (1990) y Fernández-Jalvo et al. (2016); la digestión es incluso mucho menor en los incisivos que permanecen en sus respectivas mandíbulas: así, de 51 incisivos inferiores solo en $1(2 \%)$ hay huellas de digestión débil según los grados distinguidos por dichos autores (o.c.). Este grado de digestión correspondería a la categoría 1, en la que la digestión es ausente o mínima, según Andrews (1990) y Fernández-Jalvo et al. (2016), y en la que dichos autores sitúan como potenciales predadores aves rapaces nocturnas como Tyto alba (la lechuza común), Asio otus (el búho chico) y Asio flammeus (el búho campestre) que generalmente cazan en medio abiertos.

En cuanto a la herpetofauna, desde el punto de vista tafonómico Sanchiz \& Martin (2011) concluyen que algunos taxones no presentan señales de alteración digestiva y podrían proceder de depredación sin ingestión o más probablemente de muerte accidental en la cueva, mientras que la rana bermeja presenta señales de digestión muy leves que podrían ser atribuibles a la lechuza común. Estos datos concuerdan con la escasa alteración digestiva observada en los restos de micromamíferos.

\section{Interpretación paleoambiental}

Por lo que respecta a las inferencias paleoambientales, por sus preferencias ecológicas, los micromamíferos identificados en El Sidrón se pueden distribuir en los siguientes biotopos: Bosque y termófilo: Apodemus, Clethrionomys glareolus, Glis glis y Eliomys quercinus; Bosque y/o pradera húmeda: Sorex y Talpa (hipogea); Ripícola: Neomys fodiens; Pradera húmeda y/o zonas encharcadas o pantanosas: Microtus oeconomus; Pradera húmeda: Arvicola terrestris (hipogea) y Microtus lusitanicus; Pradera húmeda $\mathrm{y} / \mathrm{o}$ seca: Microtus agrestis, Microtus arvalis, Oryctolagus cuniculus; Alpino, rupícola heliófila: Chionomys nivalis y Marmota marmota; Cavernícola y termófila: Rhinolophus euryale-Rhinolophus mehelyi. Los más abundantes en conjunto son los taxones propios de medio abiertos (Tabla 1 y Figuras 6 y 7), generalmente de praderas húmedas con cierto desarrollo de la vegetación a nivel del suelo. Sin embargo, aunque poco abundantes, hay cuatro taxones termófilos e indicadores de cierto desarrollo del bosque o de zonas arbóreas, y, aunque no es termófilo propiamente, Oryctolagus cuniculus es una especie que abunda en climas mediterráneos, actualmente prácticamente ausente de Asturias en dónde se localiza el yacimiento. Un medio ripícola estrictamente está indicado por Neomys fodiens. Por otra parte, Chionomys nivalis y Marmota marmota, más que especies asociadas estrictamente a un medio alpino y frío, pueden encontrar en éste los medios abiertos que les son propicios, con menos vegetación la primera y de praderas herbáceas las segunda. Esto en cuanto al yacimiento en su conjunto. Con respecto a las distintas zonas y niveles, no se pueden hacer mayores precisiones por su escasez de restos y representación faunística. Hay que tener en cuenta el modelo de relleno general, cuyos procesos no son los habituales en una serie deposicional normal, que tiene evidentes implicaciones en la organización y carácter de las evidencias incluidas en su interior. En la zona E/F/G/H/4-10 (Tabla 1), con el NMI de micromamíferos más elevado del yacimiento (26), se puede decir algo similar a lo expuesto anteriormente: la abundancia de especies de medios abiertos (Sorex araneus-Sorex coronatus, Talpa sp., Arvicola terrestris, Microtus arvalis-Microtus agrestis, Microtus lusitanicus y Oryctolagus cuniculus) de praderas 


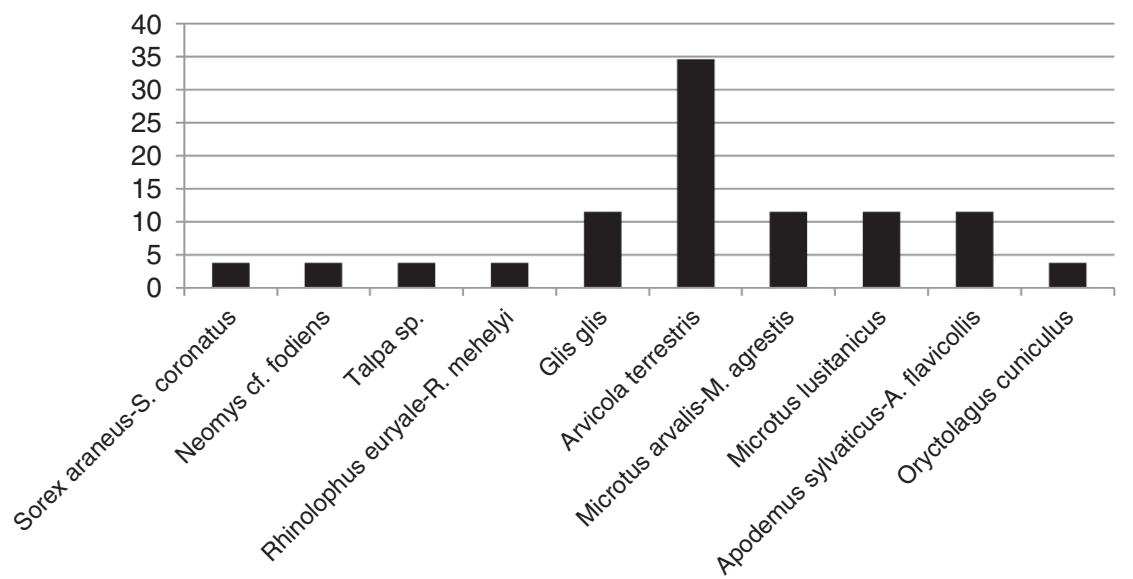

Fig. 6.- Representación gráfica de la abundancia de los taxones de micromamíferos de la zona E/F/G/H/4-10 del yacimiento de la cueva de El Sidrón según el porcentaje del NMI.

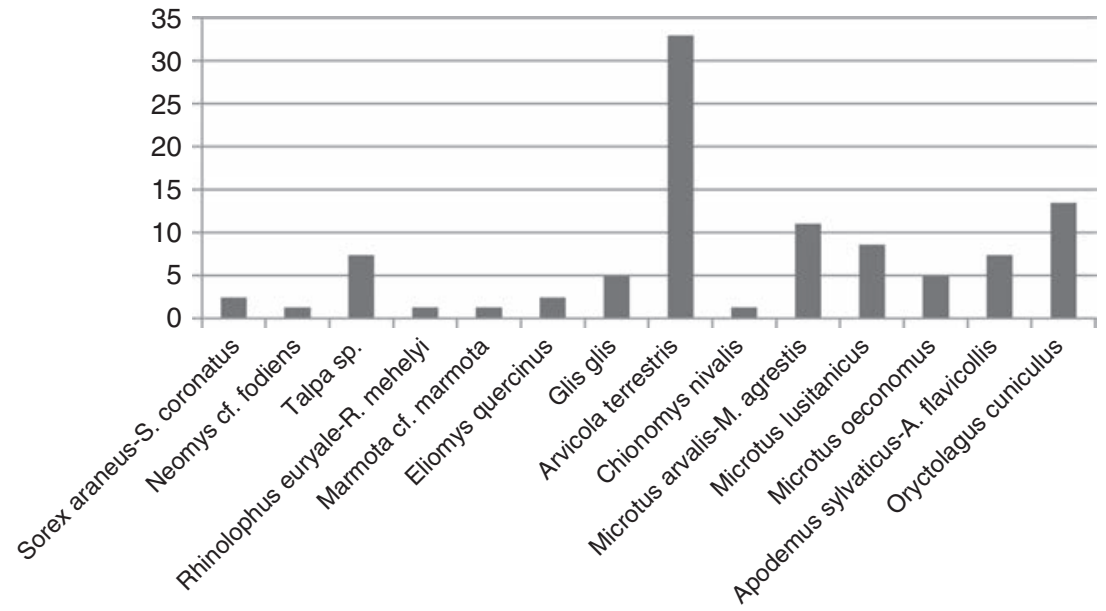

Fig. 7.-Representación gráfica de la abundancia de los taxones de micromamíferos del conjunto de sectores, zonas y niveles del yacimiento de la cueva de El Sidrón según el porcentaje del NMI. No se incluye Clethrionomys glareolus al no tener un contexto estratigráfico seguro.

húmedas por lo general con cierto desarrollo vegetal, con algunas zonas boscosas (Glis glis y Apodemus sylvaticus-Apodemus flavicollis) y un curso de agua cercano (Neomys cf. fodiens).

Es decir que, aunque los micromamíferos en general indicarían una predominancia de los espacios abiertos, generalmente de praderas de cierta humedad y desarrollo de la vegetación a nivel del suelo, habría también en el entorno zonas boscosas o arboladas de cierta entidad y algún curso de agua. El hecho de que las aves rapaces que pueden estar en el origen de las acumulaciones de restos de micromamíferos en este yacimiento cacen sus presas por lo general en medios abiertos, podría ser una de las causas de la escasez de restos de este tipo de especies forestales de las que sin embargo hay cierta variedad.

En cuanto a la temperatura, hay varios indicadores al respecto: la representación de varias especies termófilas en la asociación como se ha indicado anteriormente, aunque con escasos restos quizás por la razón anteriormente señalada, la presencia de Oryctolagus cuniculus, una especie abundante en climas templados o mediterráneos y casi ausente en la actualidad en la zona, y la ausencia de especies que indiquen un clima riguroso. En este sentido, Marmota marmota, muy escasa en restos, aunque 
actualmente es una especie alpina y heliófila, durante el Pleistoceno en la península ibérica se localiza en yacimientos situados a una altura moderada o baja, lo que hace suponer que no era una especie montana sino ligada a un hábitat de praderas, tipo de hábitat que encuentra actualmente en zonas herbáceas que abundan por encima del área forestal alpina. Todo ello hace suponer que el clima podría ser templado y relativamente húmedo, probablemente similar al actual de la zona. Estas indicaciones para el conjunto de zonas y niveles del yacimiento se mantienen para la zona E/F/G/H/4-10 mencionada anteriormente en la que hay varios indicadores termófilos (Glis glis y Apodemus sylvaticus - Apodemus flavicollis). Por otra parte Sanchiz \& Martin (2011), del estudio preliminar de la herpetofauna de El Sidrón deducen que las condiciones climáticas podrían ser algo más frías y húmedas, sobre todo en invierno, aunque señalan que no diferirían significativamente del clima actual.

\section{Conclusiones}

La asociación de micromamíferos identificada en el yacimiento de El Sidrón en este trabajo es la siguiente: Sorex araneus-Sorex coronatus, Neomys cf. fodiens, Talpa sp., Rhinolophus euryale-Rhinolophus mehelyi, Marmota cf. marmota, Eliomys quercinus, Glis glis, Arvicola terrestris, Chionomys nivalis, Microtus arvalis-Microtus agrestis, Microtus lusitanicus, Microtus oeconomus, Clethrionomys glareolus, Apodemus sylvaticus-Apodemus flavicollis y Oryctolagus cuniculus.

Todos estos taxones viven actualmente en la península ibérica y en Asturias excepto Microtus oeconomus que se extinguió en épocas históricas y Marmota marmota que se extinguió entre el final del Pleistoceno Superior y el Holoceno, aunque ha sido reintroducía a mediados del siglo XX en los Pirineos. En cuanto a Oryctolagus cuniculus, aunque actualmente es muy común en casi toda la península ibérica, está prácticamente ausente de Asturias excepto en unos pocos enclaves puntuales. El grado de digestión de los restos corresponde a la categoría 1 producida por aves rapaces nocturnas (entre las que están la lechuza, el búho chico y búho campestre), en la que la digestión es ausente o mínima según Andrews (1990) y Fernández-Jalvo et al. (2016). La asociación faunística indica la predominancia de los espacios abiertos, con praderas de cierta humedad y desarrollo de la vegetación a nivel del suelo, aunque habría también zonas boscosas o arboladas de cierta entidad, y algún curso de agua. El clima podría ser templado y relativamente húmedo, probablemente similar al actual de la zona.

\section{AGRADECIMIENTOS}

Nuestro agradecimiento a Javier Fortea (1946-2009), que lamentablemente falleció en 2009, quien, junto con uno de los autores de este trabajo (MR), amablemente ofreció a uno de nosotros (CS) participar en el proyecto de El Sidrón y estudiar los micromamíferos, a Enrique Soto por la puesta a punto de los dibujos y figuras, a Laura Ramírez, Ester Moreno y Almudena Estalrrich por el triado del sedimento para obtener los restos de microfauna, a todos los arqueólogos y paleontólogos de El Sidrón que lavaron el sedimento y triaron gran parte del material in situ, al editor de la revista, José María Cebriá, por su amabilidad, y a los revisores, César Laplana y Javier Murelaga, por su valiosas sugerencias al manuscrito. Este trabajo se ha realizado en el marco del Convenio Consejería de Cultura del Principado de Asturias/Universidad de Oviedo (CN-00-184-D3, CN-01-132-133-134-B1, CN-04-152, CN-07-088, CN-08-085, CN-09-084, SV-PA-11-14), y dentro de este entre la Universidad de Oviedo y el MNCN-CSIC, la Universidad de Alicante y la Universidad de Salamanca.

\section{Referencias}

Alcaraz-Castaño, M.; Alcolea-González, J.; Kehl, M.; Albert, R.-M.; Baena-Preysler, J.; de BalbínBehrmann, R.; Cuartero, F.; Cuenca-Bescós, G.; Jiménez-Barredo, F.; López-Sáez, J.A.; Piqué, R.; Rodríguez-Antón, D.; Yravedra, J.\& Weniger, G.-C. (2017). A context for the laste Neandertals of Interior Iberia: Los Casares cave revisited. PLoS ONE, 12 (7): e0180823

Altuna, J. (1972). Fauna de mamíferos de los yacimientos prehistóricos de Guipúzcoa. Con catálogo de los Mamíferos Cuaternarios del Cantábrico y del Pirineo Occidental. Munibe, 1-4: 464 pp.

Andrews, P. (1990). Owls, Caves and Fossils. The University of Chicago Press. $231 \mathrm{pp}$.

Arribas, O. (2004). Fauna y paisaje de los Pirineos en la Era Glaciar. Lynx, 540 pp.

Baena Preysler, J.; Carrión, E.; Ruiz, B.; Ellwood, B.; Sesé, C.; Yravedra, J.; Jordá, J.; Uzquiano, P.; Velázquez, R.; Manzano, I.; Sánchez-Marco, A. \& Hernández, F. (2005): Paleoecología y comportamiento humano durante el Pleistoceno Superior en la comarca de Liébana: La secuencia de la Cueva de El Esquilleu (Occidente de Cantabria, España). In: Neandertales Cantábricos, estado de la cuestión. Monografía del Museo Nacional y Centro de Investigación de Altamira, 
(Montes Barquín, R. \& Lasheras Corruchaga, J.A., Eds.), 20: 461-487.

Bañuls Cardona, S.; López-García, J.M.; Blain, H.-A. \& Canals Salomó, A. (2012). Climate and landscape during the Last Glacial Maximum in southwestern Iberia: The small-vertebrate association from the Sala de las Chimeneas, Maltravieso, Extremadura. Comptes Rendus Palevol, 11: 31-40.

Blanco, J.C. (1998a). Mamíferos de España: I: Insectívoros, Quirópteros, Primates y Carnívoros de la de la península Ibérica, Baleares y Canarias. Planeta, $457 \mathrm{pp}$.

Blanco, J.C. (1998b). Mamíferos de España II: Cetáceos, Artiodáctilos, Roedores y Lagomorfos de la península Ibérica, Baleares y Canarias. Planeta, 383 pp.

Cañaveras, J. C.; Sánchez-Moral, S.; Lario, J.; Cuezva, S.; Fernández, A. \& Muñoz, Ma . C. (2011). El modelo de relleno, o cómo llegaron los restos a la Galería del Osario. La cueva de El Sidrón (Borines, Piloña, Asturias). Investigación interdisciplinar de un grupo neandertal (Rasilla, M. de la; Rosas, A.; Cañaveras, J. C. \& Lalueza-Fox, C. eds.). Excavaciones Arqueológicas en Asturias, Monografías I, Consejería de Cultura y Turismo. Oviedo: 43-63.

Cañaveras; J. C.; Sánchez-Moral, S.; Cuezva, S.; Fernández Cortés, A.; Muñoz, C.; Silva, P. G.; Santos, G.; Duarte, E.; Santamaría, D. \& Rasilla, M. de la (2018). Estudio geoarqueológico de la Cueva de El Sidrón (Asturias). Boletín del Instituto Geológico y Minero, 129 (1): 107-128.

Chaline, J. (1972). Les Rongeurs du Pléistocène Moyen et Supérieur de France. (Systématique, Biostratigrahie, Paléoclimatologie). Cahiers de Paleontologie, C.N.R.S., 410 pp.

Chaline, J.; Baudvin, H.; Jammot, D. \& Saint Girons, M.C. (1974). Les proies des rapaces. Petits Mammifères et leur environnement. Doin, Paris, 141 pp.

Cuenca-Bescós, G.; Straus, L.G.; González-Morales. R. \& García-Pimienta, J.C. (2008). Paleoclima y paisaje del final del Cuaternario en Cantabria: Los pequeños mamíferos de la cueva del Mirón (Ramales de la Victoria). Revista Española de Paleontología, 23 (1): 91-126.

Delgado Castilla, L.; Pascual Molina, A. \& Ruiz Bustos, A. (1993). Geology and micromammals of the Serra-1 site (Taberas Basin, Betic Cordillera). Estudios Geológicos, 49: 361-366. https://doi.org/10.3989/egeol. 93495-6363

Duarte, E.; Santamaría, D.; Forcelledo, E.; Tarriño, A. \& Rasilla, M. de la (2016). El sílex como recurso mineral en la Prehistoria de Asturias. Cuadernos de Prehistoria y Arqueología de la Universidad de Granada, 26: 11-28.

Fernández-Jalvo, Y.; Andrews, P.; Denys, C.; Sesé, C.; Stoetzel, E.; Marin-Monfort, D. \& Pesquero, D. (2016). Taphonomy for taxonomists: Implications of predation in small mammal studies. Quaternary Science
Reviews, 139: 138-157. https://doi.org/10.1016/j. quascirev.2016.03.016

Fortea. J.; Rasilla, M. de la; Martínez, E.; Sánchez-Moral, S.; Cañaveras, J.C.; Cuezva, S.; Rosas, A.; Soler, V.; Castro, J.; Torres, T. de; Ortiz, J.E.; Julià, R.; Badal, E.; Altuna, J. \& Alonso, J. (2003). La Cueva de El Sidrón (Borines, Piloña, Asturias). Primeros resultados. Estudios Geológicos, 59: 159-179.

Fortea Pérez. J.; Rasilla Vives, M. de la; Martínez García, E.; Sánchez-Moral, S.; Cañaveras Jiménez, J.C.; Cuezva Robleño, S.; Rosas González, A.; Soler Javaloyes, V.; Castro Almazán, J.; Torres, T. de; Ortiz, J.E.; Julià, R.; Badal García, E.; Altuna, J. \& Alonso Peña, J. (2007). La Cueva de El Sidrón (Borines, Piloña, Asturias). Campañas arqueológicas de 2000 a 2002. Excavaciones Arqueológicas en Asturias 1999-2002, 5: 191-205.

Fortea, J.; Rasilla, M. de la; D. Santamaría, D.; Martínez, L.; Duarte, E.; Fernández de la Vega, J.; Martínez; E.; Cañaveras; J.C.; Sánchez-Moral, S.; Cuezva, S.; Lario, J.; Rosas, A.; Martínez-Maza, C.; GarcíaTabernero; A.; Bastir, M.; Huguet, R.; Estalrrich, A.; García-Vargas, S.; Sánchez-Meseguer, A.; León, S.; Lalueza-Fox; C.; Torres, T. de; Ortiz, J.E.; Julià, R.; Grün, R.; Valladas, H.; Mercier, N.; Tisnèrat-Laborde, N.; Soler, V.; Silva, P.G.; Carrasco, P.; Ayarza, P.; Álvarez, F.; Santos, G.; Altuna, J.; Badal, E. \& Alonso, J. (2009). La Cueva de El Sidrón (Borines, Piloña, Asturias). Campañas arqueológicas de 2003 a 2006. Excavaciones Arqueológicas en Asturias 2003-2006, 6: $367-384$

Fortea, J.; Rasilla, M. de la; Santamaría, D.; Martínez, L.; Duarte, E. \& Fernández de la Vega, J. (2010). El Paleolítico Superior en Asturias en los albores del siglo XXI. Jornadas Internacionales sobre el Paleolítico Superior Peninsular. Novedades del siglo XXI. Homenaje al Prof. Javier Fortea. Barcelona: 271-289.

García-Ibaibarriaga, N. (2015). Los microvertebrados en el registro arqueopaleontológico del País Vasco: cambios climáticos y evolución paleoambiental durante el Pleistoceno Superior. Tesis Doctoral. Universidad del País Vasco: 340 pp.

García-Ibaibarriaga, N.; Murelaga Bereikua, X.; Bailon, S.; Rofes Chávez, J. \& Ordiales Castrillo, A. (2013). Estudios de los microvertebrados de la cueva de Arlanpe (Lemoa, Bizkaia). Kobie Serie Bizkaiko Arkeologi Indusketak, 3: 81-110.

García-Ibaibarriaga, N.; Arrizabalaga, Á.; Iriarte-Chiapusso, M.J.; Rofes, J. \& Murelaga, X. (2015). The return to the Iberian Peninsula: first Quaternary record of Muscardinus and a palaeogeographical overview of the genus in Europe. Quaternary Science Reviews, 119: 106-115. https://doi.org/10.1016/j.quascirev.2015.04.017

Kowalski, K. (1992). Remarks on the zoogeography of Quaternary Rodents in Europe. Courier Forschungsinstitut Senckenberg, 153: 197-203. 
Kowalski, K. (2001): Pleistocene rodents of Europe. Folia Quaternaria, 72, 389 pp.

Lalueza-Fox. C.; Gigli, E.; Sánchez-Quinto, F.; Rasilla, M. de la; Fortea, J. \& Rosas, A. (2012a). Issues from Neandertal genomics: diversity, adaptation and hybridization revised from El Sidrón case study. Quaternary International, 247: 10-14. https://doi.org/ 10.1016/j.quaint.2010.10.012

Lalueza-Fox, C., Rosas, A. y Rasilla, M. de la. (2012b). Palaeogenetic research at the El Sidrón Neandertal site. Annals of Anatomy, 194: 133-137. https://doi. org/10.1016/j.aanat.2011.01.014

Laplana, C.; Sevilla, P.; López Martínez, N. \& Corchón Rodríguez, M.S. (2006). Primeros datos sobre los micromamíferos (Roedores, Insectívoros, Quirópteros y Lagomorfos) del Solutrense (Pleistoceno Superior final) de la Cueva de Las Caldas (Oviedo, Asturias). In: XXII Jornadas de La Sociedad Española de Paleontología y Simposios de Los Proyectos PICG 493, 503, 499, Y 467. Universidad de León: 137-139.

Laplana, C.; Blain, H.-A.; Sevilla, P.; Arsuaga, J.L.; Baquedano, E. \& Pérez-González, A. (2013). Un assemblage de petits vertébrés hautement diversifié de la fin du MIS5 dans un environnement montagnard au centre de l'Espagne (Cueva del Camino, Pinilla del Valle, Communauté Autonome de Madrid). Quaternaire, 24 (2): 207-216. https://doi.org/10.4000/quaternaire.6617

Laplana, C.; Sevilla, P.; Blain, H.-A.; Arriaza, M.C.; Arsuaga, J.L.; Pérez-González, A. \& Baquedano, E. (2016). Cold-climate rodent indicators for the Late Pleistocene of Central Iberia: New data from the Buena Pinta Cave (Pinilla del Valle, Madrid Region, Spain). Comptes Rendus Palevol, 15: 696-706. https://doi.org/10.1016/j.crpv.2015.05.010

Laplana, C.; Sevilla, P.; López Martínez, N. \& Corchón Rodríguez, M.S. (2017). Micromamíferos (Soricomorpha, Erinaceomorpha, Chiroptera, Rodentia y Lagomorpha) del Solutrense y Magdaleniense (Pleistoceno Superior final) de la Cueva de Las Caldas (Oviedo, Asturias). In: La Cueva de Las Caldas (Priorio, Oviedo). Ocupaciones magdalenienses en el Valle del Nalón (Corchón Rodríguez, M.S., Ed.). Estudios Históricos \& Geográficos, 164: 181-207

López-García, J.M. (2008). Evolución de la diversidad taxonómica de los micromamíferos en la Península Ibérica y cambios Paleoambientales durante el Pleistoceno Superior. Tesis Doctoral. Universitat Rovira I Virgili, $368 \mathrm{pp}$.

López Martínez, N. (1989). Revisión sistemática y biostratigráfica de los lagomorfos (Mammalia) del Terciario y Cuaternario de España. Memorias del Museo Paleontológico de la Universidad de Zaragoza, 3 (3): $343 \mathrm{pp}$.

Michaux, J. \& Pasquier, L. (1974). Dynamique des populations de Mulots (Rodentia, Apodemus) en Europe durant le Quaternaire. Premières données. Bulletin de la Société Géologique de France, 16 (4): 431-439.
Mitchell-Jones, A.J.; Amori, G.; Bogdanowicz, W.; Krystufek, B.; Reijnders, P.J. H.; Spitzenberger, F.; Stubbe, M.; Thissen, J.B. M.; Vohralík, V. \& Zima, J. (1999). The Atlas of European Mammals. Academic Press. T \& AD Poyser Ltd. UK, London: 484 pp.

Murelaga, X.; Sáez de Lafuente, X.; Castaños, P.; Ruiz Idarraga, R.; D’Errico, F. \& Zubeldia, H. (2007). Estudio de los micromamíferos del Pleistoceno superior de Ventalaperra (Karrantza, Bizkaia). Geogaceta, 42: 95-98.

Nicolas, V.; Martínez-Vargas, J. \& Hugot, J.P. (2016). Preliminary note: Talpa aquitania nov. sp. (Talpidae, Soricomorpha) a new mole species from southwest France and north Spain. Bulletin de l'Academie Veterinaire de France, 168 (4): 329-334. https://doi. org/10.4267/2042/58283

Palomo, L.J. \& Gisbert, J. (Eds.) (2002). Atlas de los Mamíferos terrestres de España. Dirección General de Conservación de la Naturaleza - SECEM - SECEMU, Madrid, 564 pp.

Pemán, E. (1983). Biometría y sistemática del género Neomys Kamp 1771 (Mammalia, Insectivora) en el País Vasco. Munibe, 35, 1-2: 115-148.

Pemán, E. (1985). Aspectos climáticos y ecológicos de los Micromamíferos del yacimiento de Erralla. Munibe, 37: 49-57.

Pemán, E. (1990). Los micromamíferos de la cueva de Amalda y su significación. Comentarios sobre Pliomys lenki. In: La cueva de Amalda (Zestoa, País Vasco). Ocupaciones paleolíticas y postpaleolíticas (Altuna, J., Baldeón, A. \& Mariezkurrena, K., Eds.). San Sebastián, Fundación Eusko Ikastunza: 225-238.

Pokines, J.T. (1998). The Paleoecology of Lower Magdalenian Cantabrian Spain. BAR International Series, 713: 189 pp.

Rasilla Vives, M. de la; Rosas González, A.; Cañaveras Jiménez, J.C. \& Lalueza-Fox, C. (2011). La cueva de El Sidrón (Borines, Piloña, Asturias). Investigación interdisciplinar de un grupo neandertal Excavaciones Arqueológicas en Asturias. Monografías, I, Consejería de Cultura y Turism, Oviedo, 232 pp.

Rasilla, M. de la; D. Santamaría, D.; Martínez, L.; Duarte, E.; Fernández de la Vega, J.; Suárez, P.; Díez, A.B.; Martínez; E.; Cañaveras; J.C.; Sánchez-Moral, S.; Cuezva, S.; Fernández Cortés, A.; García Antón, E.; Lario, J.; Rosas, A.; García-Tabernero; A.; Estalrrich, A.; Huguet, R.; Bastir, M.; Fernández Cascón, B.; Sesé, C.; Lalueza-Fox; C.; Silva, P.G.; Carrasco, P.; Santos, G.; Huerta, P.; Rodríguez, L.; Picón, I., Fernández, B.; Standing, M; Badal, E., Tarriño, A.; Salazar-García, D.C. \& Fortea J. (2013). La Cueva de El Sidrón (Borines, Piloña, Asturias). Campañas de excavación e investigación 2007-2012. Excavaciones Arqueológicas en Asturias 2007-2012, 7: 69-85.

Rofes, J.; Murelaga, X.; Martínez-García, B.; Bailon, S.; López-Quintana, J.C.; Guenaga-Lizasu, A.; Ortega, L.Á.; Zuluaga, M.C., Alonso-Olazabal, A., 
Castaños, J. \& Castaños, P. (2014). The long paleoenvironmental sequence of Santimamiñe (Bizkaia, Spain): 20,000 years of small mammal record from the latest Late Pleistocene to the middle Holocene. Quaternary International, 339-340: 62-75. https:// doi.org/10.1016/j.quaint.2013.05.048

Rosas, A.; Martínez Maza, C.; Bastir, M.; García-Tabernero, A.; Lalueza-Fox, C.; Huguet, R.; Ortiz, J.E.; Julià, R.; Soler, V.; Torres, T. de; Martínez, E.; Cañaveras, J.C.; Sánchez-Moral, S.; Cuezva, S.; Lario, J.; Santamaría, D.; Rasilla, M. de la \& Fortea, J. (2006). Paleobiology and comparative morphology of a late Neandertal sample from El Sidrón (Asturias, Spain). Proceedings National Academy Sciences, 103, 51: 19266-19271. https://doi.org/10.1073/pnas.0609662104

Rosas, A.; Huguet, R.; Estalrrich, A.; García-Tabernero, A.; García-Vargas, S.; Bastir, M. \& Peña-Melián, A. (2011). Fauna de macromamíferos de la Galería del Osario. La cueva de El Sidrón (Borines, Piloña, Asturias). Investigación interdisciplinar de un grupo neandertal (Rasilla, M. de la, Rosas, A., Cañaveras, J.C. y Lalueza-Fox, C. eds.). Excavaciones Arqueológicas en Asturias, Monografías I, Consejería de Cultura y Turismo. Oviedo: 147-148.

Rosas, A.; Estalrrich, A.; García-Tabernero, A.; Bastir, M.; García-Vargas, S.; Sánchez-Meseguer, A.; Huguet, R.; Lalueza-Fox, C., Peña-Melián, A.; Kranioti, E.F.; Santamaría, D.; Rasilla, M. de la \& Fortea, J. (2012). Les Néandertaliens d'El Sidrón (Asturies, Espagne). Actualisation d'un nouvel échantillon. L'Anthropologie, 116 (1): 57-76. https://doi.org/10.1016/j.anthro. 2011.12.003

Rosas, A.; Estalrrich, A.; García-Vargas, S.; GarcíaTabernero, A.; Huguet, R.; Lalueza-Fox, C., Rasilla, M. de la \& Fortea, J. (2013). Identification of Neandertal individuals in fragmentary fossil assemblages by means of teeth associations. The case of the El Sidrón (Asturias, Spain). Comptes Rendus Palevol, 12 (5): 279-291. https://doi.org/10.1016/j.crpv.2013.06.003

Rosas, A.; Estalrrich. A.; García-Tabernero, A.; Huguet, R.; Lalueza-Fox. C.; Ríos, L.; Bastir, M.; FernándezCascón, B.; Pérez-Criado, L.; Rodríguez-Pérez, F.; Ferrando, A.; Cerezo, S.; Sierra, E. y Rasilla, M. de la. (2015). Investigación paleoantropológica de los fósiles neandertales de El Sidrón (Asturias, España). Cuaternario y Geomorfología, 29: 77-94. https://doi. org/10.17735/cyg.v29i3-4.40066

Saint-Girons, M.C. (1972). La reconnaissance des proies contenues dans les pelotes de réjection des rapaces en France, Belgique, Pays-Bas et Luxembourg. Overdruk publicaties van het natuurhistorisch Genootschap in Limburg, Reeks XXII, afflevering, 1(2), 3.

Sánchez-Moral, S.; Cañaveras, J.C.; Lario, J.; Cuezva, S.; Silva, P.G.; Rasilla, M. de la \& Fortea, J. (2007). Caracterización del relleno sedimentario de la Galería del Osario (Cueva de El Sidrón, Asturias, España). XII Reunión Nacional de Cuaternario
(Lario, J. \& Silva, P.G. eds.). AEQUA, Ávila (junio 2007): 123-124.

Sanchiz, B, \& Martin, C. (2011). La herpetofauna del yacimiento de El Sidrón. In: La cueva de El Sidrón (Borines, Piloña, Asturias). Investigación interdisciplinar de un grupo neandertal (Rasilla Vives de la, M.; Rosas González, A.; Cañaveras Jiménez, J.C. \& Lalueza-Fox, C., Eds.), Excavaciones Arqueológicas en Asturias. Monografías, I, Consejería de Cultura y Turismo. Oviedo: 155-157.

Santamaría, D. (2006). Clasificación y estudio de los materiales musterienses de la Cueva de El Sidrón. Trabajo de Investigación. Inédito. Universidad de Oviedo.

Santamaría, D. (2012). La transición del Paleolítico medio al superior en Asturias. El abrigo de La Viña (La Manzaneda, Oviedo) y la cueva de El Sidrón (Borines, Piloña). Tesis doctoral, Servicio de Publicaciones de la Universidad de Oviedo. http://hdl. handle.net/10651/19328

Santamaría, D.; Fortea, F.J.; Rasilla, M. de la; Martínez, L.; Martínez, E.; Cañaveras, J.C.; Sánchez-Moral, S.; Rosas, A.; Estalrrich, A.; García-Tabernero, A. \& Lalueza-Fox. C. (2010). The technological and typological behaviour of a Neanderthal group from El Sidrón cave (Asturias, Spain). Oxford Journal of Archaeology, 29 (2): 119-148. https://doi.org/ 10.1111/j.1468-0092.2010.00342.x

Santamaría, D.; Martínez, L.; Duarte, E.; Cañaveras, J.C.; Rosas, A.; Sánchez-Moral, S.; Silva, P.G.; LaluezaFox, C.; Rasilla, M. de la. (2011). Los remontajes líticos musterienses de la Cueva de el Sidrón (Borines, Piloña, Asturias). XIII Reunión Nacional de Cuaternario (Turu, V. \& Constante, A. eds.). El Cuaternario en España y áreas afines, avances en 2011. AEQUA - Fundación Marcel Chevalier, Andorra (julio, 2011): 229-233.

Santos Delgado, G., Silva Barroso, P.G., Standing, M., Huerta, P., Fernández Macarro, B., Cañaveras Jiménez, J.C., Sánchez-Moral, S. \& Rasilla Vives, M. de la. (2012a). Análisis topográfico y geomorfológico del complejo kárstico de El Sidrón (Piloña, Asturias). VIII Congreso Nacional de Geología, (Oviedo, 2012). Geo-Temas, 13: 224.

Santos Delgado, G., Martínez Rubio, J., Silva Barroso, P.G., Sánchez-Moral, S.; Cañaveras Jiménez, J.C. \& Rasilla Vives, M. de la. (2012b). Contribución al conocimiento de la Cueva de El Sidrón (Piloña, Asturias) con técnicas de láser escáner 3D. Avances de la Geomorfología en España 2010-2012 (GonzálezDíez, A. coord. \& González-Díez, A. et al., eds.). XII Reunión Nacional de Geomorfología (Santander, 2012). 2012. PUbliCan - Ediciones de la Universidad de Cantabria: 255-258.

Sesé, C. (1994): Paleoclimatical interpretation of the Quaternary small mammals of Spain. Geobios, 27, 6: 753-767. https://doi.org/10.1016/S0016-6995(94) 80060-X 
Sesé, C. (2005): Aportación de los micromamíferos al conocimiento paleoambiental del Pleistoceno Superior de la Región Cantábrica: Nuevos datos y síntesis. In: Neandertales Cantábricos, estado de la cuestión (Montes Barquín, R. \& Lasheras Corruchaga, J.A., Eds.). Monografías del Museo Nacional y Centro de Investigación de Altamira, 20: 167-200.

Sesé, C. (2011). Los pequeños mamíferos del yacimiento de El Sidrón. In: La cueva de El Sidrón (Borines, Piloña, Asturias). Investigación interdisciplinar de un grupo neandertal (Rasilla Vives, M. de la; Rosas González, A.; Cañaveras Jiménez, J.C. \& Lalueza-Fox, C., Eds.), Excavaciones Arqueológicas en Asturias. Monografías, I, Consejería de Cultura y Turismo. Oviedo: 148-155.

Sesé, C. (2017a). Los micromamíferos (Eulipotyphla, Chiroptera, Rodentia y Lagomorpha) del yacimiento del Pleistoceno Superior de la cueva de El Castillo (Cantabria). Estudios Geológicos, 73(2): e072. http://dx.doi.org/10.3989/egeol.41589.471

Sesé. C. (2017b). Los micromamíferos (Erinaceomorpha, Soricomorpha, Rodentia y Lagomorpha) del yacimiento del final del Pleistoceno superior de la Cueva de Coímbre (Asturias, España) In: La Cueva de Coímbre (Peñamellera Alta, Asturias). Ocupaciones humanas en el valle del Cares durante el Paleolítico superior (Álvarez-Alonso, D. \& Yravedra, J. (Dirs.). Investigación y Mecenazgo. Fundación María Cristina Masaveu Peterson. Asturias: 238-259.

Sesé, C. \& Gil, E. (1988). Los micromamíferos del Pleistoceno medio del complejo cárstico de Atapuerca (Burgos). In: El hombre fósil de Ibeas y el Pleistoceno de la Sierra de Atapuerca (Aguirre, E.; Carbonell, E. \& Bermúdez de Castro, J.M., Eds.). I, Junta de Castilla y León: 75-88.

Sesé, C. \& Sevilla, P. (1996): Los micromamíferos del Cuaternario peninsular español: cronoestratigrafía e implicaciones bioestratigráficas. Revista Española de Paleontología, No Extraordinario: 278-287.

Sesé, C. \& Soto, E. (1988): Los micromamíferos (Rodentia, Insectivora y Lagomorpha). In: La Cueva de Ambrosio (Almería, Spain) y su posición cronoestratigráfica en el Mediterráneo Occidental (Ripoll López, S., Ed.). BAR, International Series, 462 (I): 157-168.

Sevilla, P. (1988). Estudio paleontológico de los Quirópteros del Cuaternario español. Paleontologia i Evolució. 22: 113-233.
Sevilla, P.; Laplana, C.; Sesé, C.; Arsuaga, J.L.; Baquedano, E.; Cacho Quesada, C. \& Vega-Toscano, L.G. (2011). Evidence of the presence of the Root Vole (Microtus oeconomus) in Central Spain during the Late Pleistocene. XVIII INQUA Congress, Berna, Póster.

Silva, P.G., Santos, G., Carrasco, P., Huerta, P., Ayarza, P., Álvarez Lobato, F., Fernández Macarro, B. \& Standing, M. (2011). La geomorfología, topografía y prospección geofísica del complejo de El Sidrón. La búsqueda del lugar de procedencia de los restos fósiles. La cueva de El Sidrón (Borines, Piloña, Asturias). Investigación interdisciplinar de un grupo neandertal (Rasilla, M. de la, Rosas, A., Cañaveras, J.C. \& Lalueza-Fox, C. eds.). Excavaciones Arqueológicas en Asturias, Monografías I, Consejería de Cultura y Turismo. Oviedo: 65-79.

Tarriño, A.; Duarte, E.; Santamaría, D.; Martínez, L.; Fernández de la Vega, J.; Suárez, P.; Rodríguez, V.; Forcelledo, E. \& Rasilla, M. de la. (2013). El Sílex de Piloña. Caracterización de una nueva fuente de materia prima en la Prehistoria de Asturias. Javier Fortea Pérez. Universitatis Ovetensis Magister. Estudios en Homenaje (Rasilla, M. de la, Coord.). Ediciones de la Universidad de Oviedo - Ménsula Ediciones Oviedo: 115-132.

Torres, T. de; Ortiz, J.E.; Grün, R; Eggins, S.; Valladas, H.; Mercier, N.; Tisnérat-Laborde, N.; Julià, R.; Soler, V.; Martínez, E.; Sánchez-Moral, S.; Cañaveras, J.C.; Lario, J.; Badal, E.; Rosas, A.; Santamaría, D.; Rasilla, M. de la \& Fortea, J. (2010). Dating of the hominid (Homo neanderthalensis) remains accumulation from El Sidrón cave (Borines, Asturias, North Spain): an example of multi-methodological approach to the dating of Upper Pleistocene sites. Archaeometry, 52: 680-705.

Wilson, D.E. \& Reeder, D.M. (2005). Mammal Species of the World. A Taxonomic and Geographic Reference. The Johns Hopkins University Press, Baltimore, $2142 \mathrm{pp}$.

Wood, R.; Higham, T.F.H.; Torres, T. de; TisnératLaborde, N., Valladas, H., Ortiz, J.E., Lalueza- Fox, C., Sánchez-Moral, S., Cañaveras, J.C., Rosas, A., Santamaría, D. \& Rasilla, M. de la. (2013). A new date of the Neanderthals from El Sidrón Cave (Asturias, Northern Spain). Archaeometry, 55: 148-158. https://doi.org/10.1111/j.1475-4754.2012.00671.x

Zubeldia, H.; Murelaga, X.; Bailon, S.; Aguirre, M. \& Sáez de Lafuente, X. (2007). Microvertebrados del Pleistoceno Superior del yacimiento de Antoliñako Koba (Gautegiz-Arteaga, Bizkaia). Kobie, 27: 5-49. 